\title{
Prognosis and management of cryptococcal meningitis in patients with human immunodeficiency virus infection
}

This article was published in the following Dove Press journal:

Neurobehavioral HIV Medicine

16 June 2012

Number of times this article has been viewed

\author{
Joshua Rhein \\ David R Boulware \\ Division of Infectious Diseases and \\ International Medicine, Department \\ of Medicine, University of Minnesota, \\ Minneapolis, MN, USA
}

\begin{abstract}
Cryptococcal meningitis has emerged as one of the most frequent and deadly opportunistic infections in patients with human immunodeficiency virus (HIV). Cryptococcosis has become the most common cause of adult meningitis in many parts of Africa, where it now rivals tuberculosis in all-cause mortality. While expanding access to antiretroviral therapy in many resource-limited settings has led to improvements in long-term prognosis, early mortality from HIV-associated cryptococcal meningitis remains unacceptably high. Successful management of cryptococcal meningitis is often hindered by unsatisfactory antifungal regimens or by poor control of elevated cerebrospinal fluid pressures. Immune reconstitution inflammatory syndrome also frequently complicates cryptococcal meningitis in patients with acquired immune deficiency syndrome (AIDS) initiating antiretroviral therapy, and the optimal timing of antiretroviral therapy remains unclear. The optimal initial regimen of amphotericin B and flucytosine is difficult to administer and is often not available in resource-limited settings, where cryptococcal meningitis is most prevalent. Fluid and electrolyte coadministration with amphotericin B is essential to minimizing iatrogenic comorbidities. Fortunately, several new approaches have been leading the way toward improving care for cryptococcal meningitis patients in resource-limited settings. Innovative trials utilizing different combinations of antifungal therapy are reviewed, and we summarize the efficacy of different induction regimens. Universal cryptococcal antigen screening of AIDS patients, combined with new point-of-care testing, has the potential to improve the early diagnosis of cryptococcal meningitis markedly in resource-limited settings.

Keywords: human immunodeficiency virus, acquired immune deficiency syndrome, cryptococcal meningitis, immune reconstitution inflammatory syndrome, antiretroviral therapy, antifungal therapy
\end{abstract}

\section{Introduction}

Cryptococcal meningitis is an invasive fungal infection of the central nervous system caused by the encapsulated yeast, Cryptococcus neoformans. The major predisposing factor in the development of cryptococcosis is profound cell-mediated immune deficiency, seen most commonly with advanced human immunodeficiency virus (HIV) infection. Though recognized a century ago, Cryptococcus became a major human pathogen with the emergence of the acquired immune deficiency syndrome (AIDS) epidemic. ${ }^{1,2}$ Whereas the introduction and increased availability of initially triazolebased antifungal therapy ${ }^{3}$ and later combination HIV antiretroviral therapy has decreased the incidence of cryptococcal meningitis in many high-income countries, ${ }^{4,5}$ the incidence of and mortality related to cryptococcal meningitis remain exceedingly high worldwide, particularly in settings where access to antiretroviral therapy is still limited.
Correspondence: David R Boulware MTRF 3-222, 200 I 6th Street SE, Minneapolis, MN 55455, USA

Tel + I 6126259996

Fax+l6126254410

Email boulw00I@umn.edu 
The global burden of HIV-associated cryptococcal disease is estimated at nearly one million cases annually. ${ }^{6}$ While long-term survival has improved with widespread use of antiretroviral therapy in high-income countries, early mortality remains high. ${ }^{7}$ Furthermore, expanding access to antiretroviral therapy in resource-limited settings has not yet led to compelling improvements in mortality, with 10 -week mortality rates between $24 \%$ and $37 \%$, even under optimal research conditions. ${ }^{8}$, Early mortality rates are often $>50 \%$ in routine practice where access to diagnostics or medications is limited or unavailable, intracranial pressure is uncontrolled, or in settings where other barriers to the management of cryptococcal meningitis exist. ${ }^{10-13}$ The highest burden of cryptococcal meningitis is in sub-Saharan Africa, where cryptococcal meningitis is the most frequent cause of adult meningitis. ${ }^{14,15}$ Recent estimates place cryptococcal meningitis as a leading cause of death among people with AIDS, accounting for approximately $20 \%$ of all deaths among HIV-infected individuals in Africa. ${ }^{6,16,17}$ To put this into perspective, these estimates suggest that cryptococcal meningitis accounts for as many or more deaths in Africa than tuberculosis. ${ }^{6,18,19}$

\section{Presentation and prognosis of cryptococcal meningitis in HIV infection}

HIV-associated cryptococcal meningitis has a slowly progressive subacute onset, typically presenting as a meningoencephalitis in severely immunocompromised persons. Cryptococcal meningitis generally presents (about $95 \%$ ) in persons with a CD 4 count $<100$ cells $/ \mu \mathrm{L}$, and cryptococcal meningitis is the first AIDS-defining illness in the majority. ${ }^{20,21}$ In general, cryptococcal meningitis should be considered in any patient with advanced HIV infection and symptoms referable to the central nervous system. The most common symptoms encountered include fever, malaise, and headache. Stiff neck, vomiting, visual disturbances, and altered mental status are encountered less frequently initially and typically reflect more advanced disease. ${ }^{12,22}$ Altered mental status at presentation is consistently the clinical feature most strongly associated with a poor clinical outcome. ${ }^{23,24}$ Disseminated disease is more likely with concomitant cough, dyspnea, or cutaneous lesions, most often resembling molluscum contagiosum. Specific signs on physical examination are often lacking, but may include meningismus, papilledema, and cranial nerve palsies (particularly of cranial nerve VI) in advanced disease. Cranial nerve VI palsy is strongly associated with the presence of increased intracranial pressure $>20 \mathrm{~cm} \mathrm{H}_{2} \mathrm{O}^{12}$
Persons living with AIDS generally have a higher fungal burden and a greatly reduced inflammatory response as compared with non-HIV-infected persons with cryptococcal meningitis, such as organ transplant recipients, resulting in contrasting clinical, laboratory, and histopathologic features. ${ }^{25,26}$ As such, traditional markers of central nervous system inflammation, such as fever and nuchal rigidity, are often underwhelming in AIDS-related cryptococcal meningitis, usually not occurring until late in the disease course, if at all. Conversely, raised intracranial pressure, which appears to correlate with a high fungal burden, ${ }^{22,27,28}$ is a major feature in HIV-associated cryptococcal meningitis. As a result, clinical parameters associated with elevated intracranial pressure feature prominently. The lack of an inflammatory response, combined with overwhelming cryptococcosis and extensive brain parenchymal involvement, often with silent multipleorgan dissemination, also characterizes the histopathologic findings at the time of autopsy in patients who have died with cryptococcal meningitis. ${ }^{26,27}$ Based on autopsy findings and anatomical locations of paradoxical immune reconstitution inflammatory syndrome (IRIS), ${ }^{20}$ silent disseminated cryptococcosis is often the rule, rather than the exception.

While meningitis is the most common manifestation, cryptococcosis can also present without meningitis. In two cohort studies of patients presenting with pulmonary syndromes, cryptococcosis was diagnosed in $13 \%$ in Thailand by serum antigen testing and in $11 \%$ Uganda by fungal culture of bronchoalveolar lavage fluid. ${ }^{29,30} \mathrm{In}$ both of these cohorts, cryptococcosis was never clinically suspected. Pulmonary cryptococcosis is likely substantially underdiagnosed and often misdiagnosed as smear-negative tuberculosis. ${ }^{31}$ Additionally, cryptococcosis can be associated with a nondifferentiated sepsis syndrome. In a prospective multisite sepsis study in Uganda, C. neoformans was the fourth most common microorganism isolated from aerobic bacterial blood cultures in persons presenting with sepsis criteria. ${ }^{32}$

The sepsis and pneumonia studies are illustrative in that cryptococcosis was an incidental unexpected finding, which is often the case when one is not specifically pursuing cryptococcal diagnostics. These observations also lend further support to a pathogenic model of primary cryptococcosis occurring in sequential, though often overlapping, stages, whereby inhalation of yeast cells or spores ${ }^{33}$ leads to an initial pulmonary infection, followed by fungemia, and finally seeding of the meninges. This model is further supported by trials demonstrating a high prevalence $(34 \%-39 \%)$ of pneumonia by chest imaging on initial presentation. ${ }^{34,35}$ Furthermore, concomitant pulmonary involvement is an independent risk factor for mortality in 
cryptococcal disease. ${ }^{35,36}$ In a large retrospective analysis in the US, for example, respiratory failure, pneumonia, and syncope were all strongly associated with inpatient mortality. ${ }^{35}$

\section{Diagnosis}

The laboratory diagnosis of cryptococcal meningitis is generally straightforward, though in practice early diagnosis can be hindered by the insidious nature of cryptococcosis, the overall lack of specific early symptoms, lack of knowledge of HIV status, and empiric treatment for possible alternative diagnoses, eg, malaria, tuberculosis, and pneumonia. The high fungal burden usually seen in HIV-associated cryptococcal meningitis results in characteristic cerebrospinal fluid findings, and analysis of the cerebrospinal fluid with India ink staining usually (approximately 80\%-85\%) suggests the diagnosis. In cases of suspected cryptococcal meningitis, lumbar puncture with measurement of the opening pressure is therefore of the utmost urgency.

\section{Cerebrospinal fluid parameters}

The initial opening pressure is elevated $\left(>20 \mathrm{~cm} \mathrm{H}_{2} \mathrm{O}\right)$ in about $70 \%$ of patients in Africa and $50 \%-60 \%$ in the US. ${ }^{12,22,37}$ The India ink stain can be used to identify encapsulated yeasts on direct microscopic examination of cerebrospinal fluid. This stain accentuates the polysaccharide capsule of C. neoformans, and is $80 \%-85 \%$ sensitive in HIV-related cryptococcal meningitis. ${ }^{38}$ There is a characteristic lack of cerebrospinal fluid pleocytosis (usually $<50$ white blood cells $/ \mu \mathrm{L}$ ) in AIDS patients, and up to $25 \%$ of patients may have normal white blood cell counts and normal protein levels in their cerebrospinal fluid. ${ }^{39,40}$ Furthermore, the lack of inflammatory markers in cerebrospinal fluid on initial lumbar puncture is an indicator of poor prognosis in cryptococcal meningitis. ${ }^{12,40,41}$ Glucose levels in cerebrospinal fluid can also be variably low or normal. Blood cultures can frequently be positive in cases of AIDS-associated cryptococcosis. Often this is incidental growth in aerobic bacterial blood culture or mycobacterial blood culture systems; ${ }^{42}$ however, fungemia likely reflects a minority of overall cases in comparison with cryptococcal antigen (CRAG) testing. ${ }^{43}$ The diagnosis of cryptococcal meningitis is conclusively established by isolating the organism on cerebrospinal fluid culture, yet antigen testing with results immediately available has surpassed culture as a diagnostic tool to guide initial therapy.

\section{Cryptococcal antigen}

The detection of CRAG in cerebrospinal fluid or serum is an important diagnostic tool. Commercially available tests for detection of CRAG, either by latex agglutination or by enzyme immunoassay, have been available for several years. ${ }^{44}$ The presence of CRAG in cerebrospinal fluid not only helps confirm the diagnosis, but can provide useful prognostic information. For example, initial CRAG titers $>1: 1024$ are predictive of higher acute mortality. ${ }^{41}$ Therefore, CRAG testing should always be performed on cerebrospinal fluid for all patients with HIV suspected of having meningitis, regardless of other cerebrospinal fluid parameters. A normal cerebrospinal fluid profile (with normal white blood cell and protein levels) does not exclude cryptococcal meningitis. The CRAG test is both sensitive (93\%-100\%) and specific (93\%-98\%) in cerebrospinal fluid. ${ }^{32}$ Importantly, in very early disease with a low fungal burden, the CRAG test appears to be more sensitive than culture, particularly if a small volume of cerebrospinal fluid is cultured $(<1 \mathrm{~mL}){ }^{12}$ Testing for serum CRAG is also another diagnostic option in suspected cryptococcal meningitis because the sensitivity of serum CRAG is $\geq 99 \%$ when the cerebrospinal fluid CRAG is positive. ${ }^{45}$ Serum antigenemia in HIV-infected patients with any central nervous system symptoms should prompt a lumbar puncture for further cerebrospinal fluid evaluation and treatment, particularly measurement and control of intracranial pressure. ${ }^{21,46}$ Conversely, when unexpected or discordant cerebrospinal fluid results are seen (cerebrospinal fluid CRAG positive, cerebrospinal fluid culture negative), serum CRAG testing can help confirm the diagnosis.

High initial CRAG titers generally correlate with high fungal burden by quantitative culture. However, the correlation is roughly accurate but not precise, because the CRAG titer in cerebrospinal fluid is reflective of the presence of a shed cryptococcal capsule (from viable and/or dead organisms), and this capsule shedding has strain to strain variability. The lack of CRAG titer precision has generally implied that \pm 1 dilution of the titer is considered to be equivalent. Enzyme-linked immunoassay measurement of CRAG (Immuno-Mycologics Inc, Norman, OK) is another technique utilized which can provide more precision. ${ }^{28}$ While high CRAG titers are associated with poor outcome, and the titers generally fall with successful treatment, historically titers have not been used in the management of acute cryptococcal meningitis. ${ }^{47,48}$ However, residual CRAG burden when starting antiretroviral therapy appears to be a risk factor for paradoxical IRIS. ${ }^{20,49}$

\section{Culture}

While culture is considered the gold standard for diagnosis of cryptococcal meningitis, culture lacks sensitivity for 
cryptococcosis unrelated to the central nervous system, necessitates a dependent period of incubation of typically $\geq 72$ hours, requires an adequate volume of a clinical specimen, and requires a laboratory infrastructure that is typically not present in resource-limited settings. The yeast grows readily at room temperature on Sabouraud dextrose agar and identification is not challenging. This agar is inexpensive but not always available. CRAG assays by latex agglutination or enzyme immunoassay are highly sensitive and specific, less labor-intensive and time-intensive than cultures, and can be utilized from either cerebrospinal fluid or serum specimens. Several factors prohibit the widespread use of CRAG testing in resource-limited settings, including laboratory infrastructure requiring heat inactivation of the sample and electricity, need for refrigeration of reagents, and a cold chain for reagent shipping. Cold chain shipping dramatically increases costs of what should be a relatively inexpensive CRAG latex agglutination assay (less than \$5).

\section{Lateral flow assay}

For these reasons, efforts have recently been advanced toward the development of an inexpensive point-of-care, lateral flow immunoassay, as advocated by the World
Health Organization. ${ }^{50}$ In July 2011, the US Food and Drug Administration approved a point-of-care lateral flow immunoassay for the detection of CRAG (Figure 1, Immuno-Mycologics Inc). This CRAG lateral flow immunoassay has a 10-minute processing time, is stable at room temperature, does not require cold chain shipping, and requires minimal technical expertise without the need for a centralized laboratory. Lateral flow immunoassay has performed well when compared with enzyme immunoassay in the testing of serum. ${ }^{43}$ Unlike latex agglutination, the lateral flow immunoassay can also be used for other sample types, such as plasma, urine, and possibly whole blood by finger-stick. ${ }^{43,51}$ These tests have the potential to improve the early diagnosis of cryptococcal meningitis markedly in resource-constrained settings.

\section{Treatment of cryptococcal meningitis in HIV infection}

The Infectious Diseases Society of America divides the treatment of cryptococcal meningitis into three phases, ie, a two-week induction phase, followed by an eight-week consolidation phase, and an extended maintenance phase thereafter for secondary prophylaxis. ${ }^{52}$ The guideline recommendations consider both the optimal medical therapy as
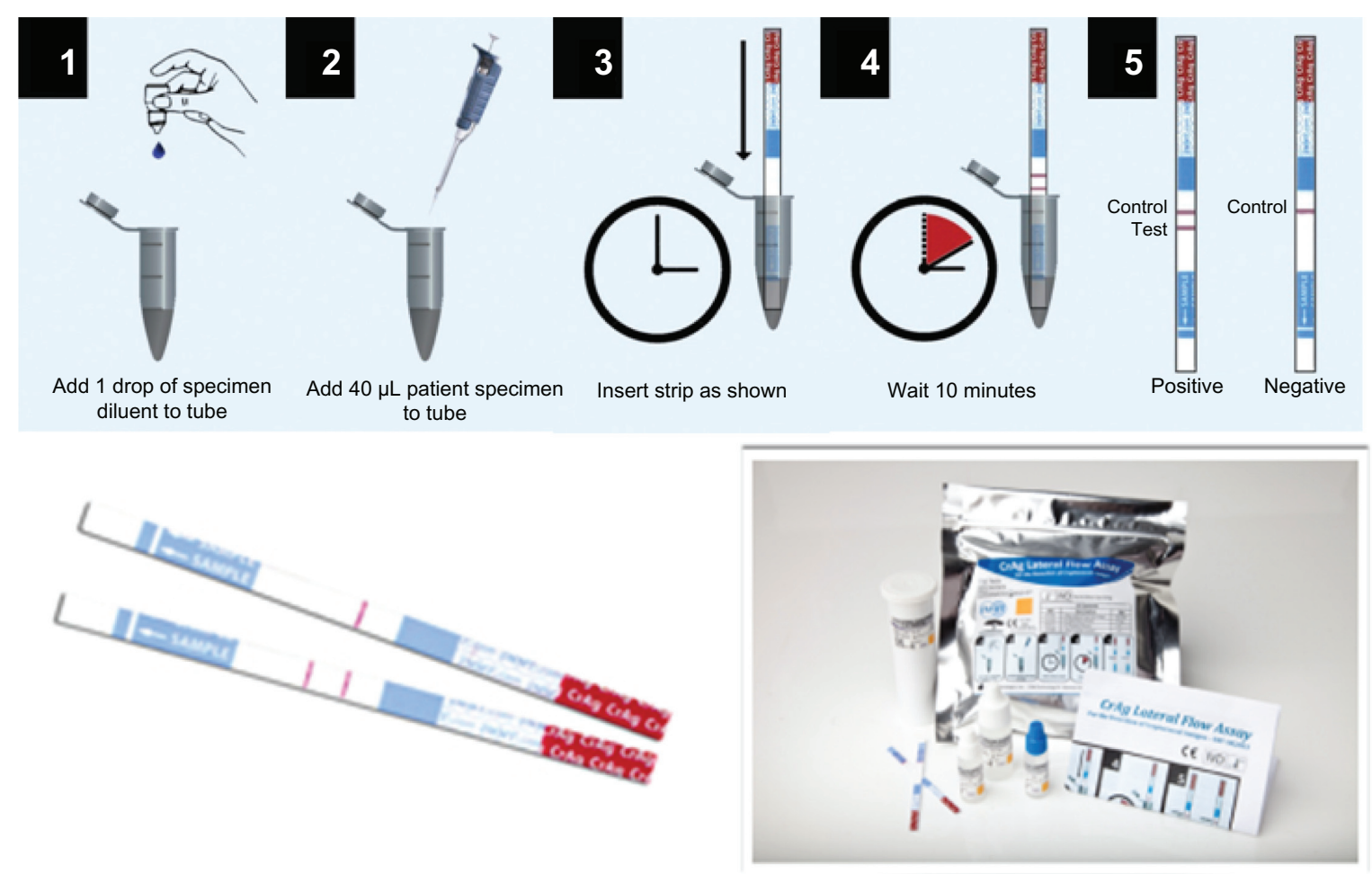

Figure I Cryptococcal antigen lateral flow assay.

Reproduced with permission by (Immuno-Mycologics Inc, Norman, OK).

Notes: This assay is a qualitative or semiquantitative (requires additional dilutions) method for detection of cryptococcal antigen in serum or cerebrospinal fluid. After combining the specimen sample with the diluent, a test strip is inserted, with the results obtained in 10 minutes. Each kit contains 50 test strips, along with the diluent and positive control. Cost from the manufacturer for resource-limited regions is $\$ 2$ per test. 
well as the management of the complications of cryptococcal meningitis frequently encountered (Table 1). Historically, international treatment guidelines have been informed primarily by large multisite clinical trials with endpoints of treatment success and survival at 2 weeks and 10 weeks.

\section{Induction therapy}

Currently recommended first-line induction therapy for cryptococcal meningitis consists of 2 weeks of amphotericin B in combination with flucytosine. This recommendation is based on the combination of amphotericin-flucytosine leading to better cerebrospinal fluid culture sterilization than amphotericin alone; however, there has never been a published mortality benefit shown with the addition of flucytosine over a series of clinical trials in the 1980s and 1990s. The first large clinical trial directly comparing amphotericin $(0.4-0.5 \mathrm{mg} / \mathrm{kg} /$ day $)$ and fluconazole (200 mg/day) demonstrated similar overall survival in the pre-antiretroviral therapy era, though there was both lower early mortality and more rapid sterilization of the cerebrospinal fluid with amphotericin as compared with fluconazole. ${ }^{53}$ Neither dosage utilized was optimal in retrospect. A second US clinical trial using higher doses of amphotericin $(0.7-0.8 \mathrm{mg} / \mathrm{kg} /$ day $)$ with or without the addition of flucytosine $100 \mathrm{mg} / \mathrm{kg} /$ day subsequently informed the currently accepted guidelines. ${ }^{54,55}$ While the combination of amphotericin and flucytosine appears to be rapidly fungicidal and may have a lower risk of treatment failure than alternative 14-day regimens, ${ }^{56,57}$ the added benefit of flucytosine as a second agent over other antifungals continues to be debated. ${ }^{58,59}$ A recent (as yet unpublished) trial from Vietnam has for the first time demonstrated superior 10 -week survival in amphotericin $(0.7-1.0 \mathrm{mg} / \mathrm{kg} /$ day $)$ plus flucytosine $(\times 2$ weeks $)$ versus amphotericin $\mathrm{B}(\times 4$ weeks $)$ alone and a trend toward better survival in comparison with amphotericin + fluconazole $800 \mathrm{mg} /$ day $\times 2$ weeks. ${ }^{60}$ This is the first trial to show a survival benefit over amphotericin alone. However, Loyse et al demonstrated that the microbiologic activity of adding fluconazole $1200 \mathrm{mg}$ /day appears to be very similar to flucytosine and at lower cost, better toxicity profile, and much greater availability in Africa. ${ }^{58}$

\section{Management with amphotericin B}

Amphotericin is well known for its frequent and often severe side effects. Significant infusion reactions must be anticipated and the tendency toward intravenous line-associated phlebitis necessitates a sizable and experienced nursing commitment over the induction phase. Nursing care is very important. Intravenous lines should be flushed after amphotericin is administered and peripheral intravenous sites rotated every 3 days to minimize thrombophlebitis. Vigorous fluid coadministration of 1-2 L saline/day, along with careful monitoring for renal dysfunction and particularly electrolyte abnormalities $\left(\mathrm{K}, \mathrm{Mg}, \mathrm{HCO}_{3}\right)$ are additional prerequisites. In otherwise relatively young subjects without pre-existing kidney disease or concomitant nephrotoxic medications and appropriate intravenous fluid administration, dosing-limited acute renal failure is actually very rare $(<5 \%)$ during 2 weeks of amphotericin administration. However, nephrotoxicity does occur without overt creatinine rise, with increasingly cumulative massive losses of potassium and magnesium beginning 5 days after amphotericin initiation. Electrolyte management with amphotericin is essential, and recognized as an essential part of the package of care in World Health Organization treatment guidelines. ${ }^{50,61}$ Whenever amphotericin B deoxycholate is administered, supplemental potassium $40-60 \mathrm{mEq} /$ day and magnesium 8-16 $\mathrm{mEq} /$ day are required, with increasing amounts in the second week of amphotericin. ${ }^{50,61}$ Without routine supplementation, the majority of persons will develop severe hypokalemia by the second week of amphotericin, which can be life-threatening.

Table I Antifungal treatment recommendations for cryptococcal meningitis in HIV-infected individuals

\begin{tabular}{|c|c|c|c|}
\hline Timeline & $\begin{array}{l}\text { Induction } \\
\text { First } 2 \text { weeks }\end{array}$ & $\begin{array}{l}\text { Consolidation } \\
\text { Next } 8 \text { weeks }\end{array}$ & $\begin{array}{l}\text { Maintenance } \\
\text { Until immune reconstitution }\end{array}$ \\
\hline Preferred regimen & $\begin{array}{l}\text { Amphotericin } B^{\mathrm{b}}(0.7-1.0 \mathrm{mg} / \mathrm{kg} \text { per day }) \text { plus } \\
\text { flucytosine }(100 \mathrm{mg} / \mathrm{kg} \text { per day })\end{array}$ & $\begin{array}{l}\text { Fluconazole } 400-800 \mathrm{mg} \\
\text { per dayc }\end{array}$ & Fluconazole 200 mg per daye \\
\hline Alternative regimens ${ }^{d}$ & $\begin{array}{l}\text { Amphotericin B ( } 0.7 \mathrm{mg} / \mathrm{kg} \text { per day) plus } \\
\text { fluconazole ( } 800-1200 \mathrm{mg} \text { per day) } \\
\text { Fluconazole ( } 1200 \mathrm{mg} \text { per day) plus flucytosine } \\
\text { (100 mg/kg per day) for } 6 \text { weeks } \\
\text { Fluconazole ( } 1200 \mathrm{mg} \text { per day) for } 10-12 \text { weeks }\end{array}$ & & \\
\hline
\end{tabular}

Notes: a CD4 cell count 100 cells $/ \mu \mathrm{L}$, and low or nondetectable viral load for 3 months with minimum of I year of antifungal therapy; ${ }^{b}$ liposomal amphotericin B (4 mg/kg per day) may be substituted, listed in order of highest recommendation top to bottom; 'some experts recommend fluconazole doses of $800 \mathrm{mg}$ per day until culture is known

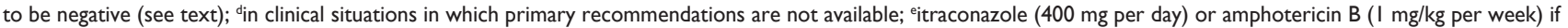
fluconazole intolerant, though inferior regimen. 
Increasing doses of amphotericin to $1 \mathrm{mg} / \mathrm{kg} / \mathrm{day}$ as compared with $0.7 \mathrm{mg} / \mathrm{kg} /$ day are more fungicidal. In the absence of underlining renal disease or clinically significant anemia, higher doses may be preferred, although no differences have been observed in mortality between these two doses. ${ }^{9}$ Liposomal and lipid complex preparations of amphotericin B are generally considered equally efficacious for treating cryptococcal meningitis and are associated with fewer adverse events and less nephrotoxicity. ${ }^{62}$ In one very small trial, more rapid cerebrospinal fluid sterilization was demonstrated with liposomal amphotericin B dosed at $4 \mathrm{mg} / \mathrm{kg}$ in comparison with $0.7 \mathrm{mg} / \mathrm{kg}$ of deoxycholate amphotericin; ${ }^{63}$ however, a larger randomized trial demonstrated no difference in efficacy. ${ }^{62}$ Because of better tolerability, lipid formulations are used more frequently in many high-income countries due to lower toxicity. These formulations are considerably more expensive and are universally unavailable in resource-limited settings.

Substantial barriers exist in adhering to the accepted guidelines in resource-limited areas, where cryptococcal meningitis is most prevalent. These include costs and availability of drugs, the requirement for intravenous administration, and the severe and potentially life-threatening toxicity of amphotericin which necessitates hospitalization and close monitoring. ${ }^{64}$ Therefore, many centers in Africa and Asia may find it difficult to adhere to recommended induction regimens. In areas where access to amphotericin is limited, for instance, centers rely on fluconazole, which is widely available via the Pfizer Diflucan Donation Program, ${ }^{65}$ for initial induction therapy. Fluconazole monotherapy is clearly a suboptimal regimen as compared with amphotericin; however, several recent trials have better informed various alternative options.

Recently, there has been a revolutionary new approach for evaluating the efficacy of cryptococcal induction therapy led by Dr. Thomas Harrison. Bicanic et al have demonstrated in a pooled series of Phase II clinical trials that the rate of cerebrospinal fluid sterilization correlates with clinical outcomes (Figure 2). ${ }^{24}$ Specifically, there is an association between early fungal activity during induction therapy and the 2-week and 10-week survival after cryptococcal meningitis. ${ }^{24}$ Quantitative culture of cerebrospinal fluid clearance is generally logarithmic over the first 2 weeks, thus the clearance can be estimated by linear regression of $\log _{10}$ colony-forming units (CFU) per mL of cerebrospinal fluid per day of therapy as a unit of measure (ie, change in $\log _{10} \mathrm{CFU} / \mathrm{mL}$ cerebrospinal fluid/day). This revelation now allows for small innovative clinical trials to estimate the early fungal activity of newer induction regimens with dramatically smaller sample sizes, using early fungal activity as a surrogate marker. Table 2 and Figure 3 summarize early fungal activity data from several recent trials comparing the early fungicidal activity of different induction regimens.

Early trials using fluconazole monotherapy at standard doses of 200-400 mg per day resulted in universally

\section{Microbiologic rate of CSF cryptococcal clearance association with survival}

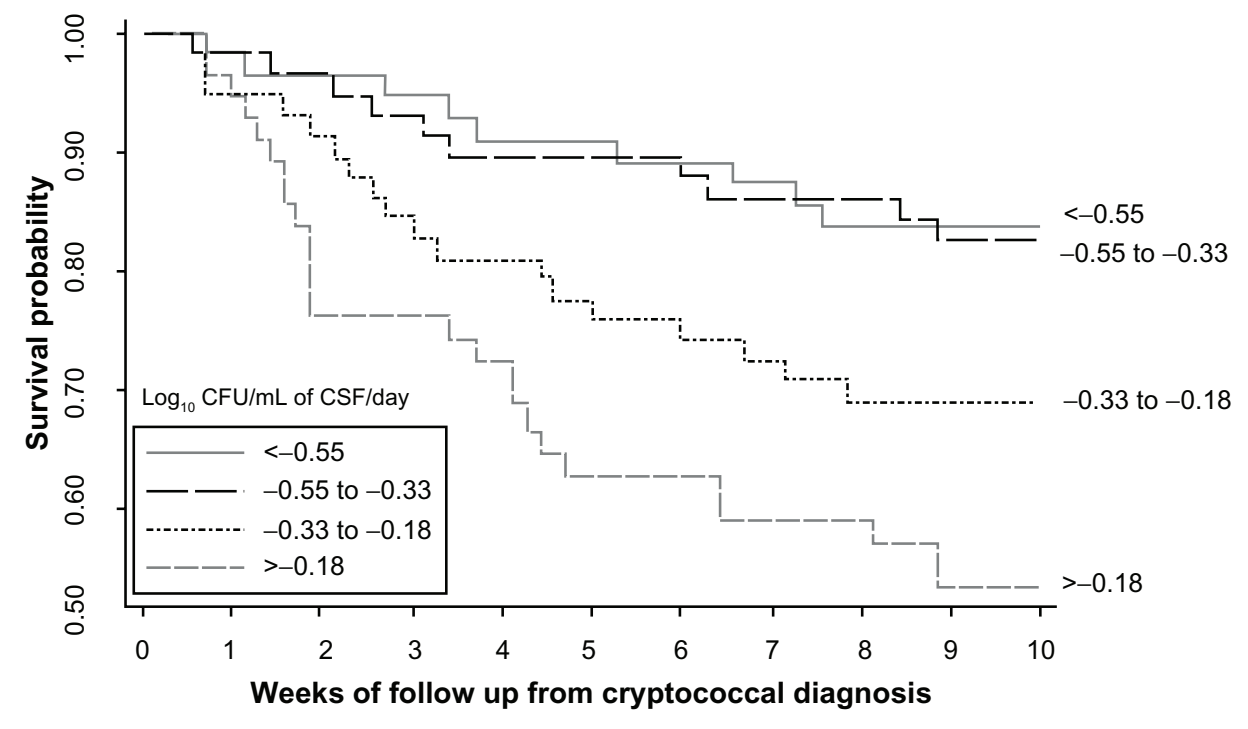

Figure 2 Kaplan-Meier survival curve by rate of clearance of infection.

Notes: More rapid clearance, measured by early fungicidal activity (measured in $\log _{10} \mathrm{CFU} / \mathrm{mL}$ of cerebrospinal fluid/day), is associated with improved early survival. Patients in top two quartiles for rate of clearance (early fungicidal activity $>0.33 \log _{10} \mathrm{CFU} / \mathrm{mL} /$ day) had a higher survival probability than patients in lower quartiles.

Abbreviations: CFU, colony-forming units; CSF, cerebrospinal fluid.

Copyright (C) 2009, Oxford Journals. Modified with permission from Bicanic T, Muzoora C, Brouwer AE, et al. Independent association between rate of clearance of infection and clinical outcome of HIV associated cryptococcal meningitis: analysis of a combined cohort of 262 patients. Clin Infect Dis. 2009;49(5):702-709. ${ }^{24}$ 
Table 2 Trials comparing early fungicidal activity of induction treatment regimens for cryptococcal meningitis

\begin{tabular}{|c|c|c|c|c|}
\hline Induction Regimen & EFA & $\pm \mathbf{S D}$ & $\mathbf{n}$ & Source \\
\hline$A m B+5 F C$ & -0.41 & 0.22 & 21 & \multirow{4}{*}{58} \\
\hline AmB + fluc (800 mg daily) & -0.38 & 0.18 & 22 & \\
\hline AmB + fluc (I 200 mg daily) & -0.41 & 0.35 & 23 & \\
\hline $\mathrm{AmB}+$ voriconazole & -0.44 & 0.20 & 13 & \\
\hline AmB (5 days) + fluc ( 1200 mg daily) & -0.30 & 0.11 & 30 & 71 \\
\hline$A m B+5 F C$ & -0.49 & NA & 30 & \multirow{2}{*}{79} \\
\hline$A m B+5 F C+I N F-\gamma$ & -0.64 & NA & 60 & \\
\hline $5 F C$ + fluconazole (I 200 mg daily) & -0.28 & 0.17 & 21 & \multirow{2}{*}{74} \\
\hline Fluconazole (I 200 mg daily) & -0.11 & 0.09 & 20 & \\
\hline AmB (7 days) + fluc ( 1200 mg daily) & -0.39 & 0.20 & 19 & \multirow{2}{*}{73} \\
\hline $\mathrm{AmB}(7$ days $)+$ fluc $(1200 \mathrm{mg}$ daily $)+5 \mathrm{FC}$ & -0.49 & 0.15 & 18 & \\
\hline$A m B(0.7 \mathrm{mg} / \mathrm{kg} /$ day $)+5 F C$ & -0.45 & 0.16 & 28 & \multirow{2}{*}{9} \\
\hline $\mathrm{AmB}(\mathrm{I} \mathrm{mg} / \mathrm{kg} /$ day $)+5 \mathrm{FC}$ & -0.56 & 0.24 & 29 & \\
\hline Fluconazole (I $200 \mathrm{mg}$ daily) & -0.18 & 0.11 & 30 & \multirow{2}{*}{ II } \\
\hline Fluconazole (800 mg daily) & -0.07 & 0.17 & 30 & \\
\hline $\mathrm{AmB}(\mathrm{I} \mathrm{mg} / \mathrm{kg} /$ day $)$ & -0.48 & 0.28 & 49 & \multirow{2}{*}{68} \\
\hline Fluconazole (400 mg daily) & -0.02 & 0.05 & 5 & \\
\hline $\mathrm{AmB}(0.7 \mathrm{mg} / \mathrm{kg} / \mathrm{day})$ & -0.31 & 0.18 & 14 & \multirow{4}{*}{56} \\
\hline$A m B(0.7 \mathrm{mg} / \mathrm{kg} /$ day $)+5 F C$ & -0.54 & 0.19 & 12 & \\
\hline AmB + fluconazole (400 mg daily) & -0.39 & 0.15 & 11 & \\
\hline$A m B+5 F C+$ fluc ( 400 mg daily) & -0.38 & 0.13 & 15 & \\
\hline
\end{tabular}

Abbreviations: EFA, early fungicidal activity ( $\log \mathrm{CFU} / \mathrm{mL} C S F / d a y) ; A m B$, amphotericin B (0.7 or I mg/kg/day or as indicated); $5 \mathrm{FC}$, flucytosine $(25 \mathrm{mg} / \mathrm{kg} 4$ times daily); Fluc, fluconazole (doses indicated); Voriconazole (300 mg twice daily; $400 \mathrm{mg}$ twice on day I); INF- $\gamma$, interferon-gamma (100 $\mu \mathrm{g}$ subcutanously, 2 or 6 doses over induction period).

poor outcomes. ${ }^{66,67}$ Fluconazole at $\leq 400 \mathrm{mg} /$ day is fungistatic, confirmed in a well designed clinical trial demonstrating no change in fungal growth by serial quantitative cultures over 2 weeks with $400 \mathrm{mg}$ /day of fluconazole. ${ }^{68}$ Furthermore, the presence of high numbers of viable organisms in cerebrospinal fluid exposed to drug over an extended period could be expected to create a high selective pressure for fluconazole resistance and symptomatic relapse. ${ }^{69,70}$ However, there is a dose-dependent effect with fluconazole. In a study from Mbarara, Uganda, Longley et al showed that fluconazole was more rapidly fungicidal when administered at a dosage of $1200 \mathrm{mg}$ per day versus $800 \mathrm{mg}$ per day. ${ }^{11}$ The quantitative rate of cerebrospinal fluid clearance increases as the fluconazole dose is increased, but it is unknown if there is any benefit of increasing fluconazole beyond $1200 \mathrm{mg} /$ day. This is the subject of a current National Institutes of Health-sponsored AIDS Clinical Trial Group trial (NCT00885703). Higher doses of fluconazole were well tolerated in two Ugandan studies, ${ }^{11,71}$ which further validates safety (but not efficacy) data for using doses up to $1600 \mathrm{mg}$ per day. ${ }^{72}$ In settings which lack access to amphotericin B and flucytosine, the guidelines now recommend treating with fluconazole $1200 \mathrm{mg}$ divided daily for 12 weeks. ${ }^{50}$ Higher doses of fluconazole can induce nausea, so fluconazole doses of $800 \mathrm{mg}$ or above, should be always divided into at least twice daily to avoid this side effect. While fungicidal activity increases with the increasing fluconazole dosing to $\geq 800 \mathrm{mg} /$ day, the fungicidal activity as yet remains inferior to amphotericin-based regimens. ${ }^{73}$

Other trials have extended the use of high-dose fluconazole to various combinations of induction therapy. A randomized controlled trial in Malawi demonstrated earlier fungicidal clearance and a trend toward improved survival when flucytosine $100 \mathrm{mg} / \mathrm{kg}$ /day was added to highdose fluconazole $1200 \mathrm{mg} /$ day for 6-week oral induction therapy ${ }^{74}$ This approach has been adopted in international guidelines as an alternative recommendation in situations where amphotericin is unavailable or its administration is impossible..$^{50,52}$ However, owing to flucytosine costs and country-level licensing issues, flucytosine is rarely available in resource-limited areas.

\section{Combination amphotericin induction therapy}

The combination of amphotericin and adjunctive fluconazole is a more widely used and/or available induction regimen (Table 3). In a trial conducted in Thailand and the US, Pappas et al demonstrated better long-term outcomes for induction therapy with amphotericin plus high-dose fluconazole $800 \mathrm{mg} /$ day compared with amphotericin alone and amphotericin plus fluconazole at lower doses of $400 \mathrm{mg} /$ day. ${ }^{75}$ Amphotericin plus fluconazole $800 \mathrm{mg} /$ day is currently the preferred regimen in the absence of flucytosine and has become the standard induction therapy at many centers. ${ }^{50,52}$ Current guidelines recommend 14 days of combination therapy, followed by at least 8 weeks of consolidation therapy with fluconazole $800 \mathrm{mg} /$ day alone..$^{50,52}$

Other trials have expanded this approach to conform to local realities. In a recent prospective cohort study from Uganda, for example, short-course amphotericin $1 \mathrm{mg} / \mathrm{kg} /$ day $\times 5$ days plus high-dose fluconazole $1200 \mathrm{mg} /$ day $\times 14$ days was well tolerated with rapid clearance of infection. ${ }^{71}$ With routine potassium supplementation of $40 \mathrm{mEq} /$ day, no significant laboratory abnormalities occurred, thus this regimen can be used without laboratory monitoring. Survival in this small prospective trial was similar to that after 2 weeks of amphotericin therapy. A definitive Phase III multisite trial is to commence in 2012 to investigate short-course amphotericin in comparison with a standard 2-week course of amphotericin and an all oral regimen of fluconazole with flucytosine.

Until recently, few trials have directly compared the different amphotericin-based adjunctive combination regi- 


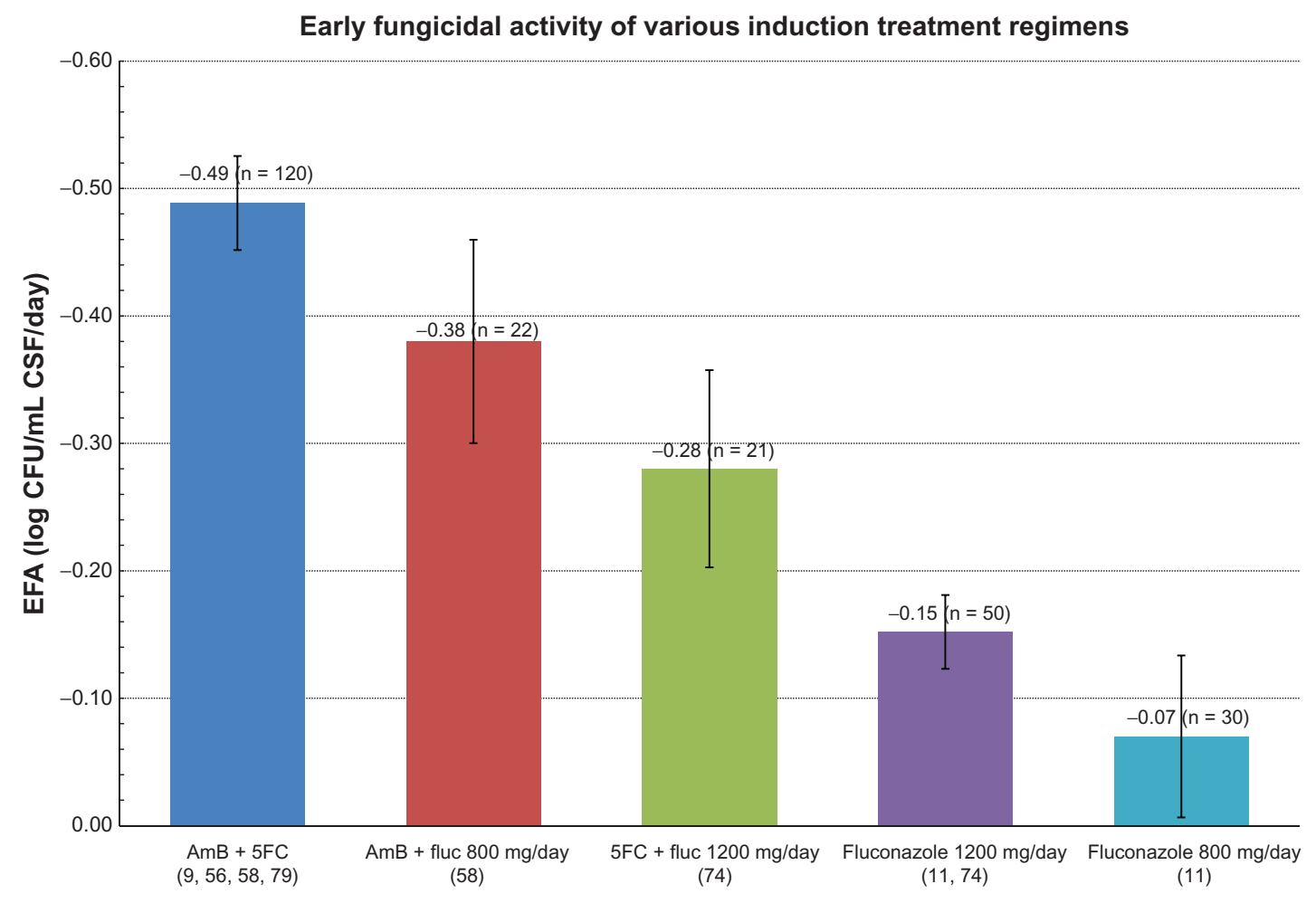

Figure 3 Early fungicidal activity of various induction regimens for cryptococcal meningitis, measured as log 10 clearance of yeasts per mL of CSF per day in quantitative CSF culture.

Notes: Values are the means with $95 \%$ confidence intervals as pooled from Table 2 (relevant citations appear in parentheses in the $\mathrm{x}$-axis). A higher negative value indicates more effective early fungicidal activity.

Abbreviations: AmB, amphotericin B; fluc, fluconazole; CFU, colony-forming units; CSF, cerebrospinal fluid; EFA, early fungicidal activity.

mens as induction therapy for HIV-associated cryptococcal meningitis. A recent randomized trial from South Africa compared four different 14-day combination induction regimens. In this trial, there were no statistically significant difference in early fungicidal activity whether subjects received adjunctive fluconazole 800 or $1200 \mathrm{mg}$ daily, flucytosine, or voriconazole in combination with amphotericin B. ${ }^{58}$ Of note, adjunctive voriconazole performed similarly to fluconazole as well as flucytosine, but offered no benefit over fluconazole for susceptible strains ( $>95 \%$ susceptible). ${ }^{76,77}$

Interferon-gamma (INF- $\gamma$ ) is another adjuvant to induction therapy. In a randomized trial conducted in the
US and Peru, patients receiving INF- $\gamma 1 \mathrm{~b}$ in addition to amphotericin \pm flucytosine induction trended toward a greater proportion having cerebrospinal fluid sterilization at 14 days versus adjunctive placebo. ${ }^{78}$ In more recent randomized trial in Cape Town, the addition of short-course INF- $\gamma 1 \mathrm{~b}$ to amphotericin plus flucytosine significantly increased early fungal activity clearance with an approximately $30 \%$ relative improvement ( -0.64 versus $-0.49 \log \mathrm{CFU} / \mathrm{mL}$ cerebrospinal fluid/day) as compared with amphotericin plus flucytosine alone..$^{79}$ The combination of amphotericin plus oral flucytosine plus subcutaneous INF- $\gamma 100 \mu \mathrm{g}$ (days 1 and 3) is the most microbiologically active induction therapy yet

Table 3 Recommended treatment for cryptococcal meningitis in resource-limited settings

\begin{tabular}{|c|c|c|c|}
\hline Medication and dose & $\sim 2$ weeks ${ }^{b}$ & 8 weeks & 52 weeks \\
\hline \multicolumn{4}{|c|}{$\begin{array}{l}\text { Amphotericin }(0.7-1.0 \mathrm{mg} / \mathrm{kg} / \text { day })+ \\
\text { second adjunctive agent } \mathrm{t}^{\mathrm{a}}\end{array}$} \\
\hline Fluconazole $800-1200 \mathrm{mg}$ daily & Continue until CSF is known sterile ${ }^{c}$ & & \\
\hline Fluconazole $400 \mathrm{mg}$ daily & & & c \\
\hline Fluconazole $200 \mathrm{mg}$ daily & & & $\begin{array}{l}\text { Continue for } \geq 12 \text { months and until } \\
C D 4>200 \text { cells } / \mu \mathrm{L} \text { for } \geq 6 \text { months }\end{array}$ \\
\hline Treatment phase & Induction & Consolidation & Secondary prophylaxis \\
\hline
\end{tabular}

Notes: a Flucytosine (5FC) $100 \mathrm{mg} / \mathrm{kg} /$ day preferred where available, otherwise fluconazole at $800-1200 \mathrm{mg} /$ day in divided doses. $\mathrm{KCl} 40-60 \mathrm{mEq} / \mathrm{day}$ should be given with amphotericin; 'optimal duration of initial induction therapy is unknown. In resource-limited regions, the cost-benefit is likely maximal for one week induction with amphotericin B at I mg/kg/day coupled with 2 weeks of fluconazole $1200 \mathrm{mg} \mathrm{kg} /$ day as the induction; 'consider longer duration of induction and consolidation therapy if CSF culture positive at 2 weeks. 
observed. Whether this translates into improved survival is not yet known.

\section{Consolidation and maintenance therapy}

After the completion of 8 weeks of consolidation therapy using fluconazole 400-800 mg/day (Tables 1 and 3), maintenance therapy with fluconazole $200 \mathrm{mg}$ daily should be initiated for long-term chronic suppression. Few trials have investigated what is optimal consolidation therapy. ${ }^{54}$ With the recognition that fluconazole $800 \mathrm{mg}$ /day is fungicidal whereas $400 \mathrm{mg} /$ day is fungistatic, the presence of sterile cerebrospinal fluid cultures and initial consolidation therapy dosing become more important considerations. The guidelines recommend repeating a lumbar puncture at 14 days of induction therapy to verify culture sterility (in addition to verifying normal intracranial pressure). Given that the day 14 cerebrospinal fluid culture typically has a low fungal burden, an incubation period of 7-10 days is often needed before cerebrospinal fluid culture with no growth is reliably negative. Thus, we recommend the continuation of fluconazole at $800 \mathrm{mg} /$ day until the 14-day cerebrospinal fluid culture is known to be negative. In effect, this is often until the patient comes back to establish ongoing therapy in the outpatient clinic 1-2 weeks later. This is an ideal time to review the 14-day cerebrospinal fluid culture and to either downwardly adjust the fluconazole dose to $400 \mathrm{mg}$ /day, or if culture-positive, to consider a repeat lumbar puncture to verify that the cerebrospinal fluid is now sterile after having received an additional few weeks of the $800 \mathrm{mg} /$ day dose of fluconazole. If suboptimal induction regimens are used, the guidelines recommend continuation of fluconazole $800 \mathrm{mg} /$ day throughout consolidation..$^{50,52}$

As Figure 4 demonstrates, the probability of sterilizing the cerebrospinal fluid by day 14 of induction therapy is dependent both on the initial fungal burden and the early fungal activity of the induction regimen used. This is further influenced by the host response to infection, with a paucity of inflammation (ie, normal white blood cells and protein, and lower IFN- $\gamma$ levels in cerebrospinal fluid) being associated with worse microbiologic clearance. ${ }^{12,24,40,41}$ Eventually, this understanding may lead to better customization and personalization of cryptococcal treatment.

\section{Predicted CSF quantitative culture clearance for various induction treatment regimens}

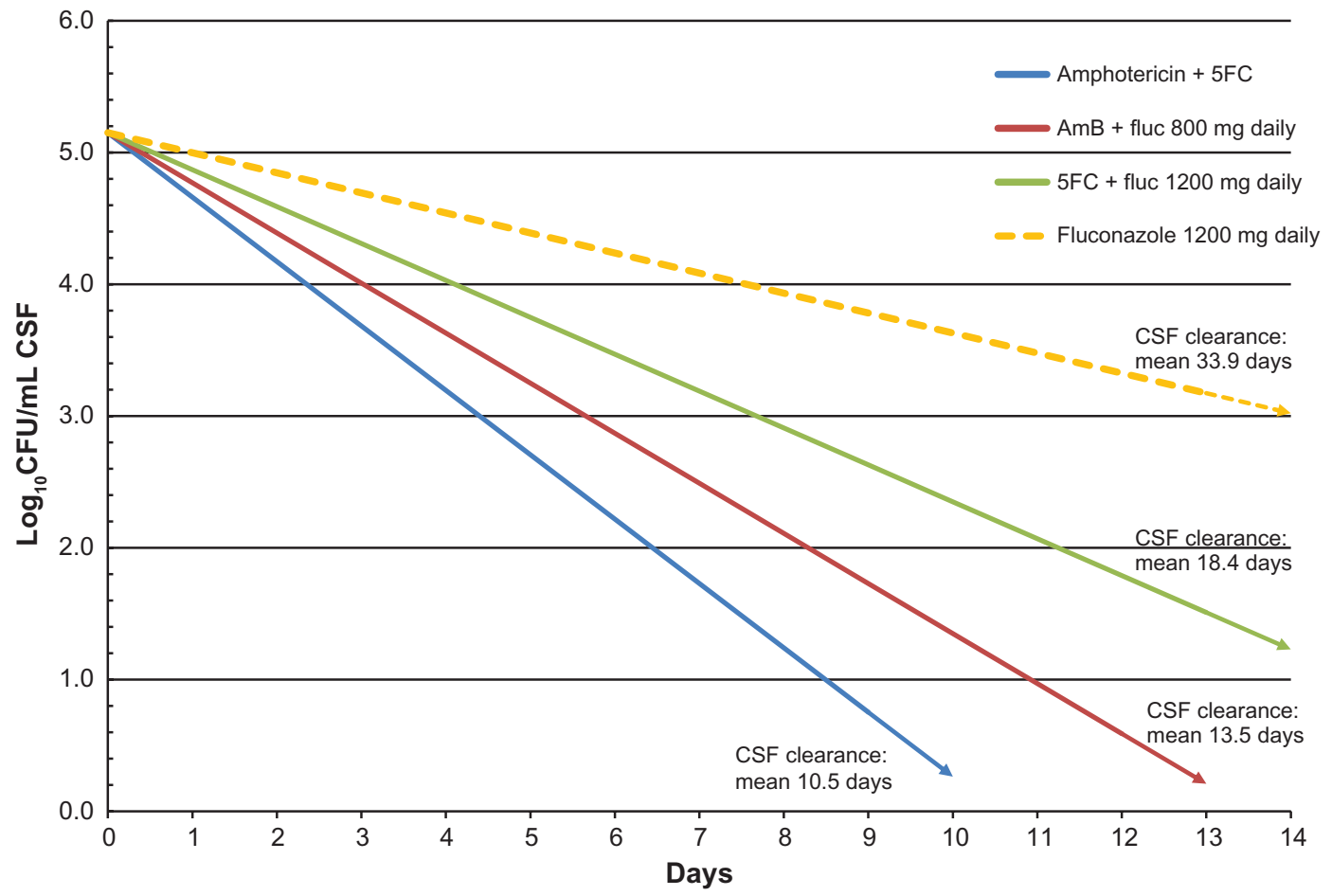

Figure 4 Predicted mean clearance of cerebrospinal fluid as a function of time for various induction regimens used for cryptococcal meningitis, based on early fungicidal activity (refer to Figure 3).

Notes: The initial cerebrospinal fluid colony count in this figure $(140,000 \mathrm{CFU} / \mathrm{mL}=5.15 \mathrm{log} \mathrm{CFU} / \mathrm{mL})$ is the median value ( $\mathrm{n}=178)$ from a large ongoing trial $(\mathrm{Cryptococcal}$ Optimal ART Timing Trial, NCT0I075152). As demonstrated, the average time required for cerebrospinal fluid sterilization can be predicted based on the initial quantitative culture of cerebrospinal fluid and the induction regimen selected. Note that depending on the induction regimen used and the initial fungal burden, clearance by 14 days is not always predicted. This provides the basis for either prolonged induction therapy or higher consolidation therapy (eg, fluconazole $800-1200 \mathrm{mg} /$ day).

Abbreviations: 5FC, flucytosine; AmB, amphotericin B; CSF, cerebrospinal fluid; fluc, fluconazole. 


\section{Secondary prophylaxis}

Prolonged maintenance therapy as secondary prophylaxis is based on a high relapse rate observed after primary therapy was discontinued in the pre-antiretroviral therapy era ${ }^{80}$ The results of two large clinical trials in the 1990s comparing fluconazole with amphotericin and itraconazole established fluconazole as the safest and most effective maintenance therapy. ${ }^{3,55}$ These trials did provide clinical experience with amphotericin and itraconazole as alternative maintenance therapy, though use should be reserved for high-risk patients with multiple relapses, fluconazole resistance, or intolerance to fluconazole. The biggest risk factor for relapse continues to be inadequate fluconazole maintenance therapy, including discontinuation by either the health system or patient-initiated discontinuation. ${ }^{81}$

Maintenance fluconazole therapy can be safely discontinued in patients demonstrating a strong and sustained immunologic response after initiation of antiretroviral therapy. Specific guidelines have been influenced by studies demonstrating a low risk of relapse in symptom-free patients who have completed primary therapy, have a sustained CD4 count $>100$ cells $/ \mu \mathrm{L}$ (based on two separate measurements 6 months apart), an undetectable viral load, and more than one year of antifungal treatment. ${ }^{52,82}$ In resource-limited settings, where routine viral load monitoring is not available, the World Health Organization recommends continuing maintenance therapy for at least 1 year and until a CD4 threshold of $>200$ cells $/ \mu \mathrm{L}$ has been reached..$^{50}$ After discontinuing fluconazole maintenance therapy, relapses are rare with successful immune reconstitution. Relapses can and do occur with immunologic failure and/or interruption of antiretroviral therapy ${ }^{83}$ however, so secondary prophylaxis should be reinstituted if CD4 counts fall below 100 cells $/ \mu \mathrm{L}$ or if serum CRAG titers increase by more than fourfold. Serum CRAG titers are not routinely checked, but in a person with a lack of immune recovery with antiretroviral therapy, this might be one surveillance method.

\section{Relapse}

Cryptococcal meningitis relapse is defined as a recurrence of symptoms, with recovery of viable organisms from previously sterile cerebrospinal fluid. In addition to reinstituting induction therapy, antiretroviral therapy should be optimized (or introduced if not yet initiated), and a thorough review of medicine adherence and potential drug interactions must be undertaken and addressed, if present. Reinduction therapy with high-dose amphotericin ( $1 \mathrm{mg} / \mathrm{kg} / \mathrm{day})$ for at least 7 days is often needed, followed by higher dose fluconazole
800-1200 mg/day. ${ }^{9}$ For refractory disease despite these measures, use of an alternative triazole such as voriconazole or weekly amphotericin should be considered. There are limited published data from resource-limited settings on the management of relapse.

In addition to monitoring closely for relapse, clinicians must also be vigilant for the development of IRIS in patients with cryptococcal meningitis initiating antiretroviral therapy. A patient experiencing recrudescence of symptoms without evidence of ongoing infection (ie, sterile cerebrospinal fluid) is more likely to be experiencing a case of paradoxical cryptococcal IRIS, rather than relapse, as discussed below. It is important to differentiate between IRIS versus cryptococcal meningitis relapse because the therapeutic approach differs depending on the etiology behind recurrent symptoms. Relapse and IRIS are not always mutually exclusive.

Relapse is also distinct from treatment failure, defined as persistence of infection, in which sterility of cerebrospinal fluid has never been achieved. Persistent infection should raise a concern for the development of fluconazole resistance. ${ }^{69}$ Susceptibility testing is important in this situation. A minimum inhibitory concentration of $\geq 16 \mu \mathrm{g} / \mathrm{mL}$ or a threefold increase in minimum inhibitory concentration is suggestive of fluconazole resistance, at which point alternative agents should be considered. Cutoff values for $C$. neoformans are based on minimal clinical data, though isolates with a minimum inhibitory concentration of $\leq 8 \mu \mathrm{g} / \mathrm{mL}$ are generally considered susceptible, while dose-dependent resistance should be considered with a minimum inhibitory concentration of $16-32 \mu \mathrm{g} / \mathrm{mL}$, and resistance should be assumed with a minimum inhibitory concentration of $\geq 64 \mu \mathrm{g} / \mathrm{mL} .^{84}$ The major risk factor for the development of resistance is the use of low-dose fluconazole as induction monotherapy. The fungicidal effect of amphotericin minimizes this risk, and resistance remains low in areas where widespread amphotericin-based induction therapy has been adopted. A switch from fluconazole monotherapy to amphotericin as initial treatment for cryptococcal meningitis, for example, has essentially eliminated the majority (but not all) cases of secondary fluconazole resistance. ${ }^{81}$

\section{Management of intracranial pressure}

Irrespective of which primary induction regimen is used, the control of cerebrospinal fluid pressure is of primary importance in the management of AIDS-associated cryptococcal meningitis. As discussed, elevated cerebrospinal fluid pressure is generally related to the high burden of yeast in the cerebrospinal fluid of AIDS patients, which 
in turn correlates with increased morbidity and mortality. ${ }^{22}$ The likely mechanism for the increased intracranial pressure in HIV-related cryptococcal meningitis is that a high fungal burden leads to clogging of the arachnoid villa with cryptococcal organisms and their shed polysaccharide capsules, leading to impairment of resorptive function. ${ }^{27}$ The cerebrospinal fluid opening pressure should be measured in any suspected case of cryptococcal meningitis. While it is often recommended that radiologic imaging be performed prior to lumbar puncture to avoid the risk of herniation, especially if focal neurologic signs are present, classic hydrocephalus and mass lesions are rare in HIV patients with cryptococcal meningitis. ${ }^{26}$ Elevated opening pressure on initial lumbar puncture needs to be managed promptly and aggressively by decompression. In most settings, this means serial lumbar punctures on a daily basis until the pressure has normalized $\left(<20 \mathrm{~cm} \mathrm{H}_{2} \mathrm{O}\right)$. Temporary percutaneous lumbar drains or ventriculostomy can be used in patients with persistent elevation. In patients with an initial opening pressure $>25 \mathrm{~cm}$ $\mathrm{H}_{2} \mathrm{O}$, enough volume of cerebrospinal fluid should be removed to reduce the opening pressure to $<20 \mathrm{~cm} \mathrm{H}_{2} \mathrm{O}^{52}$ In a patient with symptomatic cryptococcal meningitis in the absence of manometers, considering removing $30 \mathrm{~mL}$ of cerebrospinal fluid is reasonable..$^{22,28}$ Therapeutic lumbar punctures should be repeated daily until cerebrospinal fluid pressures have normalized.

Signs of raised cerebrospinal fluid pressure including headache, altered mental status, meningismus, papilledema, and changes in hearing or vision, should be closely monitored during this period. In patients who have a normal baseline opening pressure, a repeat lumbar puncture should be performed at completion of induction therapy to ensure cryptococcal clearance and to exclude development of increased intracranial pressure. Failure to identify or control raised cerebrospinal fluid pressure according to these guidelines may be neurologically detrimental. ${ }^{85}$ The use of medications other than antifungals to help manage increased intracranial pressure is not useful in HIV-associated cryptococcal meningitis, and should generally be avoided. A randomized trial conducted in Thailand was terminated early when severe metabolic complications developed in patients receiving acetazolamide. ${ }^{86}$ High-dose corticosteroid therapy is also ineffective in controlling elevated cerebrospinal fluid pressure, owing to the absence of inflammatory changes observed in cerebrospinal fluid in patients with AIDS. High-dose corticosteroids are not helpful for decreasing intracranial pressure and have been associated with higher mortality than in those not receiving corticosteroids in uncontrolled trials. ${ }^{37}$ This may have been an observational bias, and a new clinical trial is challenging this well established standard belief. The use of analgesia for cryptococcal meningitis headache should not be used in place of therapeutic lumbar punctures to lower intracranial pressure. Because the fundamental cause of the headache is raised intracranial pressure, treatment of the cause is more effective than ameliorating the symptoms.

\section{Immune reconstitution inflammatory syndrome}

With the increased availability of antiretroviral therapy in resource-limited areas of the world, IRIS has emerged as a major complication in AIDS patients. In cryptococcal IRIS, rapid immune restoration after initiation of antiretroviral therapy leads to an exaggerated, cell-mediated response to live or dead organisms or shed antigen. The exaggerated inflammatory response observed in IRIS is in stark contrast with primary cryptococcal meningitis, which is characterized by a lack of central nervous system inflammation. Cryptococcal IRIS can occur in two distinct scenarios, ie, "unmasking" disease and "paradoxical" IRIS. Prior case literature reports have at times blended these two scenarios as "IRIS," but they are distinct. In unmasking IRIS, symptoms first appear after initiation of antiretroviral therapy due to previously unrecognized cryptococcal infection.$^{87}$ This unmasking IRIS form can be thought of as an enhanced response to active infection upon immune reconstitution with antiretroviral therapy. In clinical practice, distinguishing unmasking IRIS from acquisition of new infection in the context of persistent immunodeficiency is impossible ${ }^{87}$ However, based on CRAG screening studies, it has become more clear that this unmasking IRIS is often unmasking of an existing subclinical, untreated cryptococcal infection in persons who have detectable CRAG in their peripheral blood when starting antiretroviral therapy. ${ }^{21,46}$ Pre-antiretroviral therapy CRAG screening and pre-emptive treatment can prevent antiretroviral therapy-associated cryptococcosis..$^{21,88}$

\section{CRAG screening and early targeted therapy}

Several recent studies have provided further evidence that systematic screening of patients with advanced AIDS with serum CRAG is a valuable strategy for the early detection of cryptococcal meningitis. ${ }^{17,21,89,90}$ Among severely immunosuppressed patients (CD4 count $<100$ cells $/ \mu \mathrm{L}$ ) initiating antiretroviral therapy in these studies, the prevalence of positive serum CRAG screenings ranged from $4 \%$ to $12 \%$ (Figure 5). ${ }^{21,91}$ Furthermore, asymptomatic cryptococcal 
antigenemia has been shown to be an independent predictor of death during the first 12 weeks in community and clinic cohorts. ${ }^{17,21,46}$ With expanding access to antiretroviral therapy in areas with a high prevalence of cryptococcal meningitis, an increasing proportion of diagnoses of cryptococcal meningitis occur among patients who have recently been started on antiretroviral therapy. ${ }^{92}$ This unmasking of subclinical cryptococcosis often occurs within 3 months among patients who had subclinical disease when starting antiretroviral therapy. ${ }^{87,93}$ For these reasons, the 2011 World Health Organization rapid advice recommends universal pre-antiretroviral therapy CRAG screening in all HIV patients with CD4 counts $<100$ cells $/ \mu \mathrm{L}$ entering HIV care. ${ }^{50}$ Long-term, 5 -year outcomes of asymptomatic CRAG-positive persons screened and pre-emptively treated have better outcomes than persons who develop symptomatic meningitis. ${ }^{94}$ The optimal dose and duration of fluconazole in this situation is unclear, though as discussed previously, higher doses are likely to be more effective. On the basis of the available data, treatment with fluconazole $800 \mathrm{mg} /$ day for 2 weeks, followed by $400 \mathrm{mg} /$ day for 8 weeks, is conditionally recommended by the World Health Organization. ${ }^{50}$ This recommendation is currently based on expert opinion.

\section{Paradoxical IRIS}

Paradoxical IRIS is a scenario in persons with cryptococcal meningitis pre-antiretroviral therapy who are treated and then whose symptoms recur after initiating antiretroviral therapy. This clinically appears as relapse, but cultures are typically sterile. Paradoxical IRIS can be thought of as an exaggerated response to dead fungal elements or persistent antigen following successful treatment of cryptococcal meningitis. Common manifestations of cryptococcal IRIS include relapsing meningitis, lymphadenitis, cutaneous lesions, pneumonitis, and cryptococcomas. ${ }^{20,95-97}$ The proportion of patients with cryptococcal meningitis that develop IRIS after initiation of antiretroviral therapy ranges from $8 \%$ to $45 \%$, and the median time of onset after initiation of antiretroviral therapy is approximately 8 weeks. ${ }^{12,20,24,97,98}$ In contrast with other forms of IRIS, in which severe manifestations are rare, cryptococcal IRIS is associated with substantial morbidity and mortality. ${ }^{20,93}$ It is important to differentiate IRIS from microbiologic relapse, other opportunistic infections, bacterial sepsis, and drug-related side effects. Given that there is no laboratory test available for detection, cryptococcal IRIS is primarily a clinical diagnosis of exclusion. In cryptococcal IRIS, the principal consideration is excluding culture-positive relapse.

Regardless of whether a diagnosis of cryptococcal IRIS can be confirmed with certainty, aggressive control of raised cerebrospinal fluid pressure and optimization of antifungal therapy should ensue as outlined above. No detailed guidelines currently exist regarding the optimal medical management of cryptococcal IRIS. Control of intracranial pressure is likely to be of major importance. In addition, and in contrast with unmasking IRIS or recurrent cryptococcal disease, paradoxical IRIS may require an anti-inflammatory management approach, rather than initiation or change in antifungal medication. Specific recommendations are lacking and management decisions must be individualized according
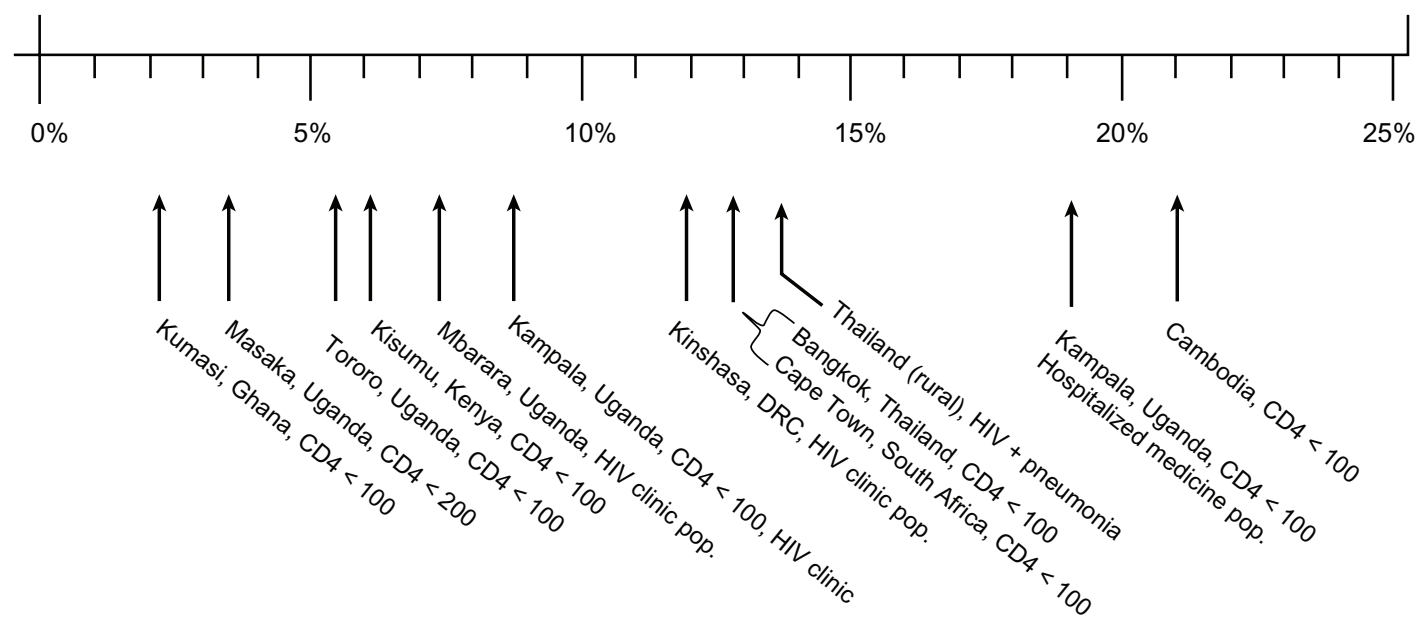

Figure 5 Cryptococcal antigen screening based on asymptomatic prevalence by location.

Notes: The reported cross-sectional prevalence rates of asymptomatic antigenemia in various outpatient clinics. Populations of HIV-infected people without a prior history of cryptococcal meningitis are displayed.

Abbreviations: ART, antiretroviral therapy.

Copyright (C) 2010, Oxford Journals. Modified with permission from Meya DB, Manabe YC, Castelnuovo B, et al. Cost-effectiveness of serum cryptococcal antigen screening to prevent deaths among HIV-infected persons with a CD4+ cell count $\leq 100$ cells/ $\mu \mathrm{L}$ who start HIV therapy in resource-limited settings. Clin Infect Dis. 20 I0;5I (4):448-455. ${ }^{21}$ 
to the presentation. Minor manifestations not involving the central nervous system often spontaneously resolve within weeks with observation. Discontinuation of antiretroviral therapy is rarely necessary. ${ }^{20,99}$ Corticosteroids, on the other hand, are at times utilized in severe cases of paradoxical cryptococcal IRIS, and several case reports have reported benefit. ${ }^{100,101}$ While no clinical trials have been conducted to validate their use in IRIS associated with cryptococcal meningitis, additional support comes from a randomized trial of prednisone in the treatment of IRIS associated with nonsevere tuberculosis. ${ }^{5,102}$ High-dose corticosteroids are safe to use in HIV-infected patients with controlled viral loads. ${ }^{103}$ However, in tropical and subtropical regions, caution is warranted, because up to $5 \%$ of patients may have chronic, asymptomatic strongyloidiasis, ${ }^{104}$ and corticosteroids may precipitate life-threatening hyperinfection. ${ }^{105}$ Thus, there may be a mortality risk of steroids without a survival benefit from treating IRIS.

\section{Optimal timing of antiretroviral therapy initiation}

The optimal time to initiate antiretroviral therapy in patients with cryptococcal meningitis is unclear. While recent trials have supported earlier initiation of antiretroviral therapy across a range of opportunistic infections (Figure 6), ${ }^{106,107}$ concerns about an increased risk of cryptococcal IRIS are often used as a rationalization for delaying antiretroviral therapy in patients with cryptococcal meningitis. ${ }^{97,100}$ Conversely, long delays in antiretroviral therapy can place patients at risk for progressing immunosuppression and mortality from com-

Favors early ART Favors deferred ART

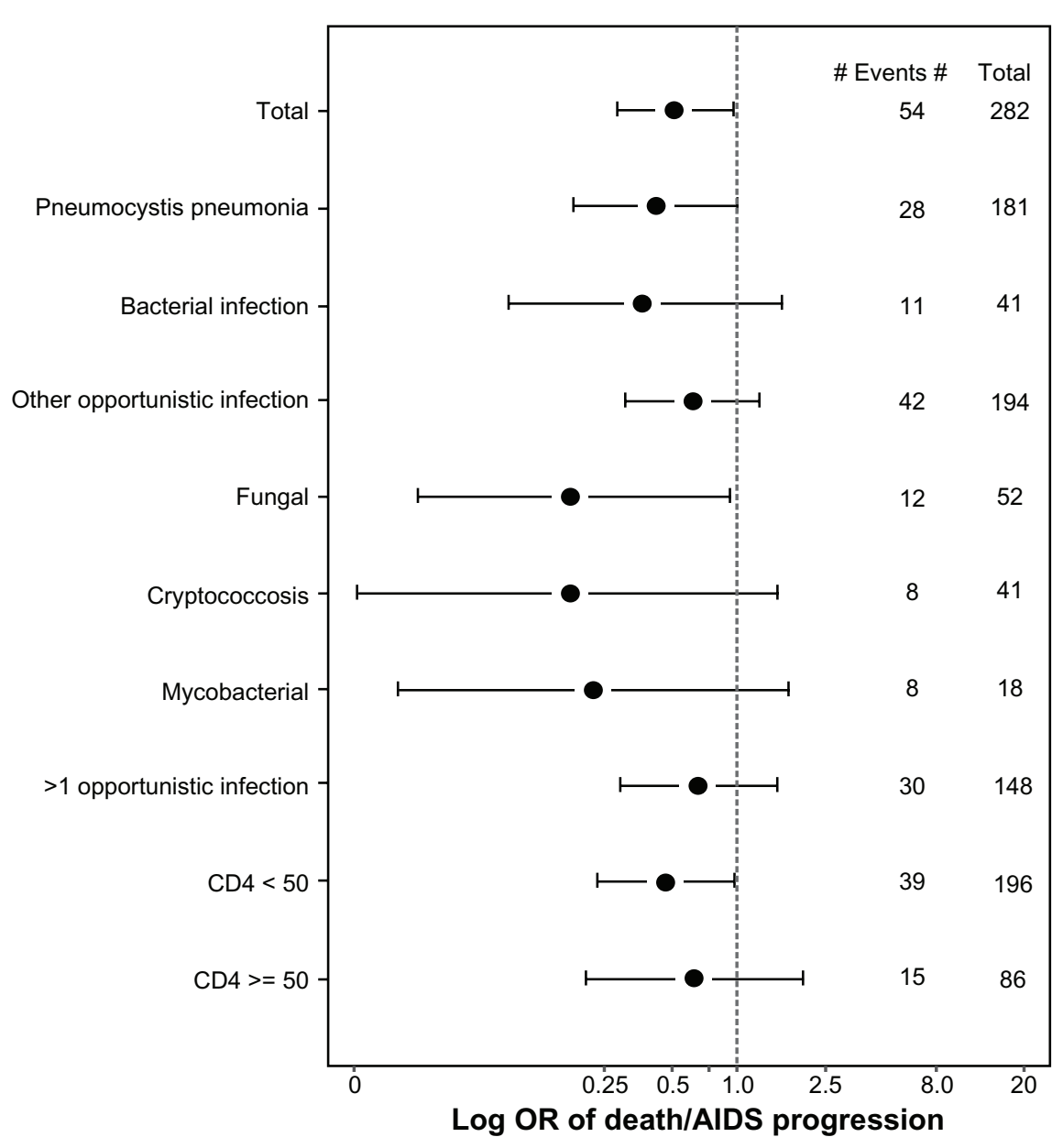

Figure 6 AIDS progression/death by opportunistic infection versus timing of initiation of ART.

Notes: Figures given as log OR, with $95 \%$ confidence intervals, with OR $<1.0$ favoring early ART versus deferred initiation of ART. Total, fungal and CD4 $<50$ categories represent significance at $P<0.05$. Fungal infections include cryptococcal infections and histoplasmosis.

Abbreviations: AIDS, acquired immunodeficiency virus; ART, antiretroviral therapy; OR, odds ratio.

Copyright (C) 2009, Public Library of Science. Reproduced from Zolopa A, Andersen J, Powderly W, et al. Early antiretroviral therapy reduces AIDS progression/death in individuals with acute opportunistic infections: a multicenter randomized strategy trial. PLoS One. 2009;4(5):e5575. ${ }^{105}$ 
plications of advanced HIV infection. In two retrospective studies, early initiation (less than 4-8 weeks after diagnosis of cryptococcal meningitis) of antiretroviral therapy was associated with an increased risk of IRIS, ${ }^{97,100}$ though this association was not observed in three more recent prospective cohorts..$^{20,24,108}$ Two randomized trials have also addressed the question of timing of antiretroviral therapy in cryptococcal meningitis patients; both used mortality as a primary endpoint in a relatively small number of subjects with cryptococcal meningitis. In the multicenter AIDS Clinical Trial Group A5164 trial, delayed initiation of antiretroviral therapy for 6 weeks was associated with increased mortality when compared with initiation of treatment at 2 weeks, supporting earlier initiation of antiretroviral therapy. ${ }^{106,109}$ The difference among the small subset of subjects with cryptococcal meningitis was not significant, though the trend favored earlier antiretroviral therapy. In a second small trial conducted in Zimbabwe, very early ( $<72$ hours) initiation of antiretroviral therapy in patients receiving fluconazole induction monotherapy was associated with increased mortality compared with a delay of 10 weeks. ${ }^{110}$ Rates of IRIS were not assessed, and the causes of death were unclear in this trial. A large multicenter trial is currently underway at African sites on the timing of antiretroviral therapy after treatment for cryptococcal meningitis (Cryptococcal Optimal Antiretroviral Therapy Timing Trial), and it is hoped that this will help to answer definitively the question of when to start antiretroviral therapy in patients with cryptococcal meningitis.

\section{Conclusion}

In summary, cryptococcal meningitis is a leading cause of morbidity and mortality in HIV-infected patients, particularly in resource-limited areas of the world. Symptoms typically begin indolently, and can include fever, malaise, and headache. Altered mental status is a marker for advanced disease and is predictor of poor outcome. Analysis of cerebrospinal fluid during primary disease is notable for a lack of inflammation in AIDS patients. The presence of cryptococcal antigen in cerebrospinal fluid or serum is central in the diagnosis of cryptococcal meningitis. New point-of-care testing has the potential to improve early diagnosis of cryptococcal meningitis in resource-limited settings markedly, and has been recommended by the World Health Organization. Updated treatment guidelines have been published that consider the optimal medical therapy in HIV-associated cryptococcal meningitis as well as complications that are frequently encountered in its management. However, substantial barriers exist in adhering to the accepted guidelines in resource-constrained areas of the world where cryptococcal meningitis is most prevalent. Fluconazole is widely available worldwide, and is an essential component through all phases of treatment, though is not preferred for induction monotherapy. Innovative trials utilizing different combinations of antifungal therapy are ongoing, and are leading the way in efforts to improve the care of HIV patients with cryptococcal meningitis, in whom IRIS has emerged as a major complication. The optimal time to initiate antiretroviral therapy in patients with cryptococcal meningitis remains unclear, and trials are underway to answer this question. Systemic screening of AIDS patients with serum CRAG is proving to be a valuable strategy for early detection and targeted treatment of cryptococcal disease.

\section{Acknowledgment}

Research support for this work was received from the National Institutes of Health, National Institute of Allergy and Infectious Diseases (K23AI073192 to DRB and T32AI055433 to JR).

\section{Disclosure}

The authors report no conflicts of interest in this work.

\section{References}

1. Hajjeh RA, Brandt ME, Pinner RW. Emergence of cryptococcal disease: epidemiologic perspectives 100 years after its discovery. Epidemiol Rev. 1995;17(2):303-320.

2. Chayakulkeeree M, Perfect JR. Cryptococcosis. Infect Dis Clin North Am. 2006;20(3):507-544.

3. Powderly WG, Saag MS, Cloud GA, et al. A controlled trial of fluconazole or amphotericin B to prevent relapse of cryptococcal meningitis in patients with the acquired immunodeficiency syndrome. The NIAID AIDS Clinical Trials Group and Mycoses Study Group. N Engl J Med. 1992;326(12):793-798.

4. Mirza SA, Phelan M, Rimland D, et al. The changing epidemiology of cryptococcosis: an update from population-based active surveillance in 2 large metropolitan areas, 1992-2000. Clin Infect Dis. 2003;36(6): 789-794.

5. Kaplan JE, Hanson D, Dworkin MS, et al. Epidemiology of human immunodeficiency virus-associated opportunistic infections in the United States in the era of highly active antiretroviral therapy. Clin Infect Dis. 2000;30 Suppl 1:S5-S14.

6. Park BJ, Wannemuehler KA, Marston BJ, Govender N, Pappas PG, Chiller TM. Estimation of the current global burden of cryptococcal meningitis among persons living with HIV/AIDS. AIDS. 2009;23(4): 525-530.

7. Lortholary O, Poizat G, Zeller V, et al. Long-term outcome of AIDSassociated cryptococcosis in the era of combination antiretroviral therapy. AIDS. 2006;20(17):2183-2191.

8. Jarvis JN, Boulle A, Loyse A, et al. High ongoing burden of cryptococcal disease in Africa despite antiretroviral roll out. AIDS. 2009;23(9): 1182-1183.

9. Bicanic T, Wood R, Meintjes G, et al. High-dose amphotericin B with flucytosine for the treatment of cryptococcal meningitis in HIVinfected patients: a randomized trial. Clin Infect Dis. 2008;47(1): 123-130. 
10. Park BJ, Shetty S, Ahlquist A, et al. Long-term follow-up and survival of antiretroviral-naive patients with cryptococcal meningitis in the preantiretroviral therapy era, Gauteng Province, South Africa. Int J STD AIDS. 2011;22(4):199-203.

11. Longley N, Muzoora C, Taseera K, et al. Dose response effect of high-dose fluconazole for HIV-associated cryptococcal meningitis in southwestern Uganda. Clin Infect Dis. 2008;47(12):1556-1561.

12. Kambugu A, Meya DB, Rhein J, et al. Outcomes of cryptococcal meningitis in Uganda before and after the availability of highly active antiretroviral therapy. Clin Infect Dis. 2008;46(11):1694-1701.

13. Wajanga BM, Kalluvya S, Downs JA, Johnson WD, Fitzgerald DW, Peck RN. Universal screening of Tanzanian HIV-infected adult inpatients with the serum cryptococcal antigen to improve diagnosis and reduce mortality: an operational study. $J$ Int AIDS Soc. 2011;14:48

14. Jarvis JN, Meintjes G, Williams A, Brown Y, Crede T, Harrison TS Adult meningitis in a setting of high HIV and TB prevalence: findings from 4961suspected cases. BMC Infect Dis. 2010;10:67.

15. Hakim JG, Gangaidzo IT, Heyderman RS, et al. Impact of HIV infection on meningitis in Harare, Zimbabwe: a prospective study of 406 predominantly adult patients. AIDS. 2000;14(10):1401-1407.

16. French N, Gray K, Watera C, et al. Cryptococcal infection in a cohort of HIV-1-infected Ugandan adults. AIDS. 2002;16(7): 1031-1038.

17. Liechty CA, Solberg P, Were W, et al. Asymptomatic serum cryptococcal antigenemia and early mortality during antiretroviral therapy in rural Uganda. Trop Med Int Health. 2007;12(8):929-935.

18. Okongo M, Morgan D, Mayanja B, Ross A, Whitworth J. Causes of death in a rural, population-based human immunodeficiency virus type 1 (HIV-1) natural history cohort in Uganda. Int J Epidemiol. 1998;27(4):698-702.

19. Corbett EL, Churchyard GJ, Charalambos S, et al. Morbidity and mortality in South African gold miners: impact of untreated disease due to human immunodeficiency virus. Clin Infect Dis. 2002;34(9):1251-1258.

20. Boulware DR, Meya DB, Bergemann TL, et al. Clinical features and serum biomarkers in HIV immune reconstitution inflammatory syndrome after cryptococcal meningitis: a prospective cohort study. PLoS Med. 2010;7(12):e1000384.

21. Meya DB, Manabe YC, Castelnuovo B, et al. Cost-effectiveness of serum cryptococcal antigen screening to prevent deaths among HIV-infected persons with a CD4+ cell count $\leq 100$ cells $/ \mu \mathrm{L}$ who start HIV therapy in resource-limited settings. Clin Infect Dis. 2010;51(4):448-455.

22. Bicanic T, Brouwer AE, Meintjes G, et al. Relationship of cerebrospinal fluid pressure, fungal burden and outcome in patients with cryptococcal meningitis undergoing serial lumbar punctures. AIDS. 2009;23(6): 701-706

23. Bicanic T, Harrison TS. Cryptococcal meningitis. Br Med Bull. 2004;72:99-118.

24. Bicanic T, Muzoora C, Brouwer AE, et al. Independent association between rate of clearance of infection and clinical outcome of HIV associated cryptococcal meningitis: analysis of a combined cohort of 262 patients. Clin Infect Dis. 2009;49(5):702-709.

25. Lee YC, Wang JT, Sun HY, Chen YC. Comparisons of clinical features and mortality of cryptococcal meningitis between patients with and without human immunodeficiency virus infection. J Microbiol Immunol Infect. 2011;44(5):338-345.

26. Klock C, Cerski M, Goldani LZ. Histopathological aspects of neurocryptococcosis in HIV-infected patients: autopsy report of 45 patients. Int J Surg Pathol. 2009;17(6):444-448.

27. Loyse A, Wainwright H, Jarvis JN, et al. Histopathology of the arachnoid granulations and brain in HIV-associated cryptococcal meningitis: correlation with cerebrospinal fluid pressure. AIDS. 2010;24(3): 405-410.

28. Wijewardana I, Jarvis JN, Meintjes G, Harrison TS, Bicanic T. Large volume lumbar punctures in cryptococcal meningitis clear cryptococcal antigen as well as lowering pressure. J Infect. 2011;63(6):484-486.
29. Harris JR, Lindsley MD, Henchaichon S, et al. High prevalence of cryptococcal infection among HIV-infected patients hospitalized with pneumonia in Thailand. Clin Infect Dis. 2012;54(5):e43-e50.

30. Deok-jong Yoo S, Worodria W, Davis JL, et al. The prevalence and clinical course of HIV-associated pulmonary cryptococcosis in Uganda. $J$ Acquir Immune Defic Syndr. 2010;54(3):269-274.

31. Jarvis JN, Wainwright H, Harrison TS, Rebe K, Meintjes G. Pulmonary cryptococcosis misdiagnosed as smear-negative pulmonary tuberculosis with fatal consequences. Int J Infect Dis. 2010;14 Suppl 3: e310-e312.

32. Jacob ST, Moore CC, Banura P, et al. Severe sepsis in two Ugandan hospitals: a prospective observational study of management and outcomes in a predominantly HIV-1 infected population. PLoS One. 2009;4(11):e7782.

33. Velagapudi R, Hsueh YP, Geunes-Boyer S, Wright JR, Heitman J. Spores as infectious propagules of Cryptococcus neoformans. Infect Immun. 2009;77(10):4345-4355.

34. Dromer F, Mathoulin-Pelissier S, Launay O, Lortholary O; French Cryptococcosis Study. Determinants of disease presentation and outcome during cryptococcosis: the CryptoA/D study. PLoS Med. 2007;4(2):e21.

35. Sajadi MM, Roddy KM, Chan-Tack KM, Forrest GN. Risk factors for mortality from primary cryptococcosis in patients with HIV. Postgrad Med. 2009;121(2):107-113.

36. Cameron ML, Bartlett JA, Gallis HA, Waskin HA. Manifestations of pulmonary cryptococcosis in patients with acquired immunodeficiency syndrome. Rev Infect Dis. 1991;13(1):64-67.

37. Graybill JR, Sobel J, Saag M, et al. Diagnosis and management of increased intracranial pressure in patients with AIDS and cryptococcal meningitis. The NIAID Mycoses Study Group and AIDS Cooperative Treatment Groups. Clin Infect Dis. 2000;30(1):47-54.

38. Khanna N, Chandramuki A, Desai A, Ravi V. Cryptococcal infections of the central nervous system: an analysis of predisposing factors, laboratory findings and outcome in patients from South India with special reference to HIV infection. J Med Microbiol. 1996;45(5): 376-379.

39. Darras-Joly C, Chevret S, Wolff M, et al. Cryptococcus neoformans infection in France: epidemiologic features of and early prognostic parameters for 76 patients who were infected with human immunodeficiency virus. Clin Infect Dis. 1996;23(2):369-376.

40. Boulware DR, Bonham SC, Meya DB, et al. Paucity of initial cerebrospinal fluid inflammation in cryptococcal meningitis is associated with subsequent immune reconstitution inflammatory syndrome. J Infect Dis. 2010;202(6):962-970.

41. Anekthananon T, Manosuthi W, Chetchotisakd P, et al. Predictors of poor clinical outcome of cryptococcal meningitis in HIV-infected patients. Int J STD AIDS. 2011;22(11):665-670.

42. Taniguchi T, Ogawa Y, Kasai D, et al. Three cases of fungemia in HIV-infected patients diagnosed through the use of mycobacterial blood culture bottles. Intern Med. 2010;49(19):2179-2183.

43. Lindsley MD, Mekha N, Baggett HC, et al. Evaluation of a newly developed lateral flow immunoassay for the diagnosis of cryptococcosis. Clin Infect Dis. 2011;53(4):321-325.

44. Temstet A, Roux P, Poirot JL, Ronin O, Dromer F. Evaluation of a monoclonal antibody-based latex agglutination test for diagnosis of cryptococcosis: comparison with two tests using polyclonal antibodies. J Clin Microbiol. 1992;30(10):2544-2550.

45. Asawavichienjinda T, Sitthi-Amorn C, Tanyanont V. Serum cyrptococcal antigen: diagnostic value in the diagnosis of AIDS-related cryptococcal meningitis. J Med Assoc Thai. 1999;82(1):65-71.

46. Jarvis JN, Lawn SD, Vogt M, Bangani N, Wood R, Harrison TS. Screening for cryptococcal antigenemia in patients accessing an antiretroviral treatment program in South Africa. Clin Infect Dis. 2009;48(7):856-862.

47. Powderly WG, Cloud GA, Dismukes WE, Saag MS. Measurement of cryptococcal antigen in serum and cerebrospinal fluid: value in the management of AIDS-associated cryptococcal meningitis. Clin Infect Dis. 1994;18(5):789-792. 
48. Aberg JA, Watson J, Segal M, Chang LW. Clinical utility of monitoring serum cryptococcal antigen (sCRAG) titers in patients with AIDSrelated cryptococcal disease. HIV Clin Trials. 2000;1(1):1-6.

49. Sungkanuparph S, Filler SG, Chetchotisakd P, et al. Cryptococcal immune reconstitution inflammatory syndrome after antiretroviral therapy in AIDS patients with cryptococcal meningitis: a prospective multicenter study. Clin Infect Dis. 2009;49(6):931-934.

50. World Health Organization. Rapid advice: Diagnosis, prevention and management of cryptococcal disease in HIV-infected adults, adolescents and children, 2011. Available from: http://www.who.int/hiv/pub/ cryptococcal_disease2011. Accessed April 3, 2012.

51. Jarvis JN, Percival A, Bauman S, et al. Evaluation of a novel point-ofcare cryptococcal antigen test on serum, plasma, and urine from patients with HIV-associated cryptococcal meningitis. Clin Infect Dis. 2011;53(10):1019-1023.

52. Perfect JR, Dismukes WE, Dromer F, et al. Clinical practice guidelines for the management of cryptococcal disease: 2010 update by the Infectious Diseases Society of America. Clin Infect Dis. 2010;50(3): 291-322.

53. Saag MS, Powderly WG, Cloud GA, et al. Comparison of amphotericin B with fluconazole in the treatment of acute AIDS-associated cryptococcal meningitis. The NIAID Mycoses Study Group and the AIDS Clinical Trials Group. N Engl J Med. 1992;326(2): $83-89$.

54. van der Horst CM, Saag MS, Cloud GA, et al. Treatment of cryptococcal meningitis associated with the acquired immunodeficiency syndrome. National Institute of Allergy and Infectious Diseases Mycoses Study Group and AIDS Clinical Trials Group. N Engl J Med. 1997;337(1): $15-21$.

55. Saag MS, Cloud GA, Graybill JR, et al. A comparison of itraconazole versus fluconazole as maintenance therapy for AIDS-associated cryptococcal meningitis. National Institute of Allergy and Infectious Diseases Mycoses Study Group. Clin Infect Dis. 1999;28(2): 291-296.

56. Brouwer AE, Rajanuwong A, Chierakul W, et al. Combination antifungal therapies for HIV-associated cryptococcal meningitis: a randomised trial. Lancet. 2004;363(9423):1764-1767.

57. Dromer F, Bernede-Bauduin C, Guillemot D, Lortholary O. Major role for amphotericin B-flucytosine combination in severe cryptococcosis. PLoS One. 2008;3(8):e2870.

58. Loyse A, Wilson D, Meintjes G, et al. Comparison of the early fungicidal activity of high-dose fluconazole, voriconazole, and flucytosine as second-line drugs given in combination with amphotericin B for the treatment of HIV-associated cryptococcal meningitis. Clin Infect Dis. 2012;54(1):121-128.

59. Bennett J. Companion drugs for amphotericin B in cryptococcal meningitis: flucytosine, fluconazole, or ... nothing? Clin Infect Dis. 2012;54(1):129-130.

60. Day JN. Combination antifungal therapy for HIV associated cryptococcal meningitis. Paper presented at the International Congress of Antimicrobial Agents and Chemotherapy. September 19, 2011; Chicago, IL.

61. Bahr N, Rolfes MAR, Musubire A, et al. The impact of routine electrolyte supplementation during amphotericin induction therapy in resource-limited settings. Paper presented at the 8th International Conference on Cryptococcus and Cryptococcosis. May 4, 2011; Charleston, SC.

62. Hamill RJ, Sobel JD, El-Sadr W, et al. Comparison of 2 doses of liposomal amphotericin B and conventional amphotericin B deoxycholate for treatment of AIDS-associated acute cryptococcal meningitis: a randomized, double-blind clinical trial of efficacy and safety. Clin Infect Dis. 2010;51(2):225-232.

63. Chen SC. Cryptococcosis in Australasia and the treatment of cryptococcal and other fungal infections with liposomal amphotericin B. J Antimicrob Chemother. 2002;49 Suppl 1:57-61.
64. Bicanic T, Wood R, Bekker LG, Darder M, Meintjes G, Harrison TS. Antiretroviral roll-out, antifungal roll-back: access to treatment for cryptococcal meningitis. Lancet Infect Dis. 2005;5(9):530-531.

65. Wertheimer AI, Santella TM, Lauver HJ. Successful public/private donation programs: a review of the Diflucan Partnership Program in South Africa. J Int Assoc Physicians AIDS Care (Chic). 2004;3(3): 74-79.

66. Schaars CF, Meintjes GA, Morroni C, Post FA, Maartens G. Outcome of AIDS-associated cryptococcal meningitis initially treated with $200 \mathrm{mg} /$ day or $400 \mathrm{mg} /$ day of fluconazole. BMC Infect Dis. 2006;6:118.

67. Mwaba P, Mwansa J, Chintu C, et al. Clinical presentation, natural history, and cumulative death rates of 230 adults with primary cryptococcal meningitis in Zambian AIDS patients treated under local conditions. Postgrad Med J. 2001;77(914):769-773.

68. Bicanic T, Meintjes G, Wood R, et al. Fungal burden, early fungicidal activity, and outcome in cryptococcal meningitis in antiretroviral-naive or antiretroviral-experienced patients treated with amphotericin B or fluconazole. Clin Infect Dis. 2007;45(1):76-80.

69. Bicanic T, Harrison T, Niepieklo A, Dyakopu N, Meintjes G. Symptomatic relapse of HIV-associated cryptococcal meningitis after initial fluconazole monotherapy: the role of fluconazole resistance and immune reconstitution. Clin Infect Dis. 2006;43(8):1069-1073.

70. Bii CC, Makimura K, Abe S, et al. Antifungal drug susceptibility of Cryptococcus neoformans from clinical sources in Nairobi, Kenya. Mycoses. 2007;50(1):25-30.

71. Muzoora CK, Kabanda T, Ortu G, et al. Short course amphotericin B with high dose fluconazole for HIV-associated cryptococcal meningitis. J Infect. 2012;64(1):76-81.

72. Anaissie EJ, Kontoyiannis DP, Huls C, et al. Safety, plasma concentrations, and efficacy of high-dose fluconazole in invasive mold infections. J Infect Dis. 1995;172(2):599-602.

73. Jackson A, Nussbaum J, Phulusa J, et al. A phase II randomised controlled trial adding oral flucytosine to high dose fluconazole, with short-course amphotericin B, for cryptococcal meningitis in Malawi. AIDS. 2012; In Press.

74. Nussbaum JC, Jackson A, Namarika D, et al. Combination flucytosine and high-dose fluconazole compared with fluconazole monotherapy for the treatment of cryptococcal meningitis: a randomized trial in Malawi. Clin Infect Dis. 2010;50(3):338-344.

75. Pappas PG, Chetchotisakd P, Larsen RA, et al. A Phase II randomized trial of amphotericin B alone or combined with fluconazole in the treatment of HIV-associated cryptococcal meningitis. Clin Infect Dis. 2009;48(12):1775-1783.

76. Govender NP, Patel J, van Wyk M, Chiller TM, Lockhart SR. Trends in antifungal drug susceptibility of Cryptococcus neoformans isolates obtained through population-based surveillance in South Africa in 2002-2003 and 2007-2008. Antimicrob Agents Chemother. 2011;55(6):2606-2611.

77. Mdodo R, Moser SA, Jaoko W, et al. Antifungal susceptibilities of Cryptococcus neoformans cerebrospinal fluid isolates from AIDS patients in Kenya. Mycoses. 2011;54(5):e438-e442.

78. Pappas PG, Bustamante B, Ticona E, et al. Recombinant interferongamma $1 \mathrm{~b}$ as adjunctive therapy for AIDS-related acute cryptococcal meningitis. J Infect Dis. 2004;189(12):2185-2191.

79. Jarvis JN, Meintjes G, Rebe K, et al. Adjunctive interferon-gamma immunotherapy for the treatment of HIV-associated cryptococcal meningitis: a randomized controlled trial. AIDS. March 20, 2012. [Epub ahead of print.]

80. Bozzette SA, Larsen RA, Chiu J, et al. A placebo-controlled trial of maintenance therapy with fluconazole after treatment of cryptococcal meningitis in the acquired immunodeficiency syndrome. California Collaborative Treatment Group. N Engl J Med. 1991;324(9): 580-584.

81. Jarvis JN, Meintjes G, Williams Z, Rebe K, Harrison TS. Symptomatic relapse of HIV-associated cryptococcal meningitis in South Africa: the role of inadequate secondary prophylaxis. $S$ Afr Med $J$. 2010;100(6):378-382. 
82. Vibhagool A, Sungkanuparph S, Mootsikapun P, et al. Discontinuation of secondary prophylaxis for cryptococcal meningitis in human immunodeficiency virus-infected patients treated with highly active antiretroviral therapy: a prospective, multicenter, randomized study. Clin Infect Dis. 2003;36(10):1329-1331.

83. Mussini C, Pezzotti P, Miro JM, et al. Discontinuation of maintenance therapy for cryptococcal meningitis in patients with AIDS treated with highly active antiretroviral therapy: an international observational study. Clin Infect Dis. 2004;38(4):565-571.

84. Aller AI, Martin-Mazuelos E, Lozano F, et al. Correlation of fluconazole MICs with clinical outcome in cryptococcal infection. Antimicrob Agents Chemother. 2000;44(6):1544-1548.

85. Shoham S, Cover C, Donegan N, Fulnecky E, Kumar P. Cryptococcus neoformans meningitis at 2 hospitals in Washington, DC: adherence of health care providers to published practice guidelines for the management of cryptococcal disease. Clin Infect Dis. 2005;40(3):477-479.

86. Newton PN, Thai le H, Tip NQ, et al. A randomized, double-blind, placebo-controlled trial of acetazolamide for the treatment of elevated intracranial pressure in cryptococcal meningitis. Clin Infect Dis. 2002;35(6):769-772.

87. Haddow LJ, Colebunders R, Meintjes G, et al. Cryptococcal immune reconstitution inflammatory syndrome in HIV-1-infected individuals: proposed clinical case definitions. Lancet Infect Dis. 2010;10(11):791-802.

88. Rajasingham R, Meya DB, Boulware DR. Integrating cryptococcal antigen screening and pre-emptive treatment into routine HIV care. J Acquir Immune Defic Syndr. 2012;59(5):85-91.

89. Micol R, Lortholary O, Sar B, et al. Prevalence, determinants of positivity, and clinical utility of cryptococcal antigenemia in Cambodian HIVinfected patients. J Acquir Immune Defic Syndr. 2007;45(5):555-559.

90. Jarvis JN, Meintjes G, Wood R, Harrison TS. Testing but not treating: missed opportunities and lost lives in the South African antiretroviral therapy programme. AIDS. 2010;24(8):1233-1235.

91. Oyella J, Meya D, Bajunirwe F, Kamya MR. Prevalence and factors associated with cryptococcal antigenemia among severely immunosuppressed HIV-infected adults in Uganda: a cross-sectional study. J Int AIDS Soc. 2012;15(1):15.

92. Jarvis JN, Meintjes G, Harrison TS. Outcomes of cryptococcal meningitis in antiretroviral naive and experienced patients in South Africa. J Infect. 2010;60(6):496-498.

93. Lawn SD, Bekker LG, Myer L, Orrell C, Wood R. Cryptococcocal immune reconstitution disease: a major cause of early mortality in a South African antiretroviral programme. AIDS. 2005;19(17):2050-2052.

94. Butler E, Meya DB, Boulware DR. Early treatment of Cryptococcal antigenemia improves long-term survival as compared with treatment of symptomatic Cryptococcal meningitis in persons with HIV/AIDS. Abstract 76.005 presented at the 2011 Global Health Conference. November 14, 2011; Montreal, Canada.

95. Jenny-Avital ER, Abadi M. Immune reconstitution cryptococcosis after initiation of successful highly active antiretroviral therapy. Clin Infect Dis. 2002;35(12):e128-e133.

96. Skiest DJ, Hester LJ, Hardy RD. Cryptococcal immune reconstitution inflammatory syndrome: report of four cases in three patients and review of the literature. J Infect. 2005;51(5):e289-e297.
97. Shelburne SA 3rd, Darcourt J, White AC Jr, et al. The role of immune reconstitution inflammatory syndrome in AIDS-related Cryptococcus neoformans disease in the era of highly active antiretroviral therapy. Clin Infect Dis. 2005;40(7):1049-1052.

98. Sungkanuparph S, Jongwutiwes U, Kiertiburanakul S. Timing of cryptococcal immune reconstitution inflammatory syndrome after antiretroviral therapy in patients with AIDS and cryptococcal meningitis. J Acquir Immune Defic Syndr. 2007;45(5):595-596.

99. Bicanic $\mathrm{T}$, Meintjes $\mathrm{G}$, Rebe $\mathrm{K}$, et al. Immune reconstitution inflammatory syndrome in HIV-associated cryptococcal meningitis: a prospective study. J Acquir Immune Defic Syndr. 2009;51(2):130-134.

100. Lortholary O, Fontanet A, Memain N, et al. Incidence and risk factors of immune reconstitution inflammatory syndrome complicating $\mathrm{HIV}$-associated cryptococcosis in France. AIDS. 2005;19(10): 1043-1049.

101. Huits RM, Bremmer R, Enting RH, Sprenger HG, van Assen S. Return of meningeal symptoms in a patient treated for cryptococcal meningitis. J Neurol. 2007;254(10):1443-1444.

102. Meintjes G, Wilkinson RJ, Morroni C, et al. Randomized placebo-controlled trial of prednisone for paradoxical tuberculosisassociated immune reconstitution inflammatory syndrome. AIDS. 2010;24(15):2381-2390.

103. Lesho E. Evidence base for using corticosteroids to treat HIVassociated immune reconstitution syndrome. Expert Rev Anti Infect Ther. 2006;4(3):469-478.

104. Brown M, Bukusuba J, Hughes P, et al. Screening for intestinal helminth infestation in a semi-urban cohort of HIV-infected people in Uganda: a combination of techniques may enhance diagnostic yield in the absence of multiple stool samples. Trop Doct. 2003;33(2):72-76.

105. Boulware DR, Stauffer WM, Hendel-Paterson BR, et al. Maltreatment of Strongyloides infection: case series and worldwide physicians-intraining survey. Am J Med. 2007;120(6):e541-e548.

106. Zolopa A, Andersen J, Powderly W, et al. Early antiretroviral therapy reduces AIDS progression/death in individuals with acute opportunistic infections: a multicenter randomized strategy trial. PLoS One. 2009;4(5):e5575.

107. Abdool Karim SS, Naidoo K, Grobler A, et al. Timing of initiation of antiretroviral drugs during tuberculosis therapy. $N$ Engl J Med. 2010;362(8):697-706.

108. Manosuthi W, Sungkanuparph S, Chottanapund S, et al. Temporary external lumbar drainage for reducing elevated intracranial pressure in HIV-infected patients with cryptococcal meningitis. Int J STD AIDS. 2008;19(4):268-271

109. Grant PM, Komarow L, Andersen J, et al. Risk factor analyses for immune reconstitution inflammatory syndrome in a randomized study of early versus deferred ART during an opportunistic infection. PLoS One. 2010;5(7):e11416.

110. Makadzange AT, Ndhlovu CE, Takarinda K, et al. Early versus delayed initiation of antiretroviral therapy for concurrent HIV infection and cryptococcal meningitis in Sub-Saharan Africa. Clin Infect Dis. 2010;50(11):1532-1538
Neurobehavioral HIV Medicine

\section{Publish your work in this journal}

Neurobehavioral HIV Medicine is an international, peer-reviewed, open access journal focusing on advances in research in HIV/ AIDS, with specific reference to the neurological, psychiatric and behavioral consequences of the disease, concomitant infections and specific antiretroviral therapy. The manuscript

\section{Dovepress}

management system is completely online and includes a very quick and fair peer-review system, which is all easy to use. Visit http://www.dovepress.com/testimonials.php to read real quotes from published authors. 